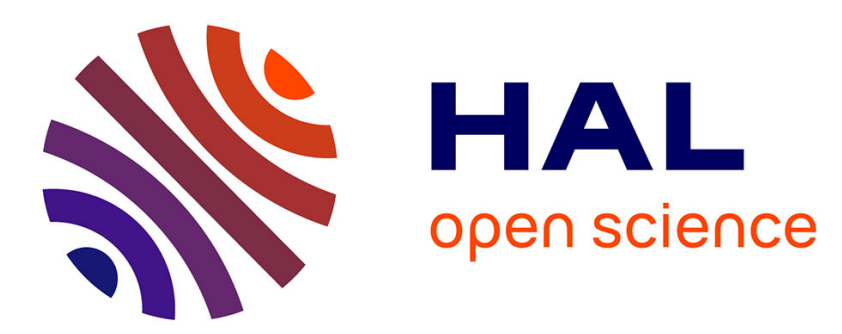

\title{
Numerical study of eigenmode forcing effects on jet flow development and noise generation mechanisms
}

\author{
Felix Keiderling, Leonhard Kleiser, Christophe Bogey
}

\section{To cite this version:}

Felix Keiderling, Leonhard Kleiser, Christophe Bogey. Numerical study of eigenmode forcing effects on jet flow development and noise generation mechanisms. Physics of Fluids, 2009, 21 (4), pp.045106. 10.1063/1.3112686 . hal-02342175

\section{HAL Id: hal-02342175 \\ https://hal.science/hal-02342175}

Submitted on 31 Oct 2019

HAL is a multi-disciplinary open access archive for the deposit and dissemination of scientific research documents, whether they are published or not. The documents may come from teaching and research institutions in France or abroad, or from public or private research centers.
L'archive ouverte pluridisciplinaire HAL, est destinée au dépôt et à la diffusion de documents scientifiques de niveau recherche, publiés ou non, émanant des établissements d'enseignement et de recherche français ou étrangers, des laboratoires publics ou privés. 


\title{
Numerical study of eigenmode forcing effects on jet flow development and noise generation mechanisms
}

\author{
Felix Keiderling, ${ }^{1, a)}$ Leonhard Kleiser, ${ }^{1}$ and Christophe Bogey ${ }^{2}$ \\ ${ }^{1}$ Institute of Fluid Dynamics, ETH Zurich, 8092 Zurich, Switzerland \\ ${ }^{2}$ Laboratoire de Méchanique des Fluides et d'Acoustique, UMR CNRS 5509, Ecole Centrale de Lyon, \\ 69134 Ecully, France
}

(Received 25 June 2008; accepted 24 February 2009; published online 23 April 2009)

\begin{abstract}
The effect of nonlinear interaction of instability eigenmodes on jet flow transition and its near acoustic field for a high-subsonic round jet at a Reynolds number of $\operatorname{Re}=4.5 \times 10^{5}$ and a Mach number of $\mathrm{Ma}=0.9$ is investigated using large-eddy simulations. At the inflow, helical perturbations of azimuthal wavenumbers $|n|=4, \ldots, 8$ determined from linear stability theory are superimposed on a laminar base flow in order to trigger transition to turbulence. The disturbance amplitude is varied parametrically in the range from $1.5 \%$ to $4.5 \%$ of the jet exit velocity $U_{j}$. Thereby we aim to characterize sources of noise generation and, in particular, underlying mode interactions. With increasing forcing amplitude, the transitional behavior of the jet changes which affects the mean flow and also the acoustic near-field, which are both analyzed in detail. As the forcing amplitude is increased, the axial root-mean-square peak levels along the jet centerline are reduced by approximately 7\%. Simultaneously, pronounced dual-peak distributions are generated along the jet lip line which are related to the localization of vortex pairings of the jet column mode. For low-amplitude excitation the azimuthal turbulent kinetic energy spectra show that the unexcited, naturally least stable axisymmetric mode $n=0$ and the helical mode $n=1$ dominate the early nonlinear regimes between $z \approx 6 r_{0}$ and $9 r_{0}$ where $r_{0}$ is the jet radius. An analysis of the Fourier mode amplitude clarifies that this energy rise is linked to the helical mode $n=1$. For higher forcing amplitudes, in addition to the varicose mode $n=0$ interactions between the excited even mode $n=4$ and higher azimuthal harmonics thereof dominate the azimuthal energy spectra. These differences in the early nonlinear development of the eigenmodes are found to alter the acoustic near-field. At small angles from the downstream jet axis, the peak acoustic frequency occurs at a Strouhal number based on the angular frequency $\omega$ and the jet diameter $D_{j}$ of $\mathrm{St}=\omega D_{j} /\left(2 \pi U_{j}\right)$ $\approx 0.4$. For low-amplitude forcing sound pressure levels are slightly enhanced which can be linked to the dominant low azimuthal wavenumbers identified in the transitional region. In the sideline direction, regardless of the excitation level, broadbanded spectra with maxima in the band 0.7 $\leq \mathrm{St} \leq 0.8$ are found which is maintained at intermediate observer angles. For high forcing amplitude, however, a tonal component outside the initially excited frequency range is observed. This peak at $\mathrm{St} \approx 0.88$ can be explained by weakly nonlinear interactions of initially forced eigenmodes $n=4$ and $n=8$ together with the jet column mode. (C) 2009 American Institute of Physics. [DOI: 10.1063/1.3112686]
\end{abstract}

\section{INTRODUCTION}

Noise prediction methods used in today's design process of jet engine nozzles largely rely on the Reynolds-averaged Navier-Stokes equations combined with acoustic analogies. ${ }^{1,2}$ These methods are well established for specific geometries, however, they remain at best semiempirical and need tuning of coefficients to particular cases in order to obtain reliable turbulence predictions. In contrast, aeroacoustic research on turbulent jets focuses on time-dependent simulation methods since the flow unsteadiness is the inherent source of sound. Direct numerical simulations (DNSs) were used for relatively low Reynolds number configurations. ${ }^{3,4}$ Far-reaching conclusions can be drawn

\footnotetext{
${ }^{a)}$ Author to whom correspondence should be addressed. Electronic mail: keiderling@ifd.mavt.ethz.ch. Telephone: +41 44632 5189. Fax: +41 44 6321147.
}

from such simulations concerning, for example, mechanisms of sound generation and the structure of noise sources. This demonstrated the applicability of direct noise computation, i.e., calculating directly the aerodynamically generated sound together with the flow in a unified approach. However, because of the high computational cost, DNS is restricted to flows in the low Reynolds number regime. At higher Reynolds numbers, as in the present work, large-eddy simulations (LES) can be employed in which only the large scales are fully resolved while the effects of subgrid scales (SGSs) are modeled. Bogey et al. ${ }^{5,6}$ Bodony and Lele, ${ }^{7,8}$ Uzun et al. ${ }^{9}$ and Andersson et al., ${ }^{10,11}$ among others, demonstrated that it is possible to directly compute the generated noise using LES.

All flow computation approaches share the problem of suitable boundary conditions at the edges of the computational domain. In the context of aeroacoustics this becomes 
even more important: as in all transitional/turbulent numerical experiments either turbulent inflow conditions are to be prescribed or disturbances have to be introduced to a laminar flow to initiate transition to turbulence. However, care has to be taken when transition is triggered artificially as this numerical noise may overwhelm the physical one. The disturbances can be either artificial or, as in the present work, based on eigenmodes of some underlying base-flow jet profile. For a survey of different treatments of the inflow boundary in the context of jet noise predictions using LES we refer to Ref. 12.

The pronounced sensitivity of jets to variations of the inflow condition has been demonstrated experimentally ${ }^{13,14}$ as well as numerically. ${ }^{15,16}$ Hence, investigating the sensitivity of the simulations to changes in disturbance amplitudes seems appropriate. The particularity of the present work lies in the nature of the disturbances introduced at the inflow. The nonlinear development of eigenmodes is believed to play an important role in the sound generation process of forced jets. ${ }^{17}$ For example, Sandham et al. ${ }^{18}$ found qualitative agreement for a model consisting of nonlinear mode interaction of a convected vortex packet with full Navier-Stokes simulation data. They reason that the wavenumber difference of disturbances and their nonlinear interaction dominate the subsonic sound radiation from eigenmodes. In contrast, Cheung et al. ${ }^{19,20}$ focus on nonlinear parabolized stability equations (PSE) and demonstrate that this approach accurately captures the acoustic near-field of subsonic jet flow (and when combined with an acoustic analogy also the far field). Only recently, Sandham and Salgado ${ }^{21}$ investigated nonlinear mode-by-mode interactions using linear PSE. Employing these modes to drive a source term of an acoustic analogy they found good agreement with experimental reference data. The latter two approaches may remain restricted to lower Reynolds numbers because the rapid amplitude growth of modes may result in convergence problems or even divergence as well as in an axially fast developing flow, which contradicts the underlying assumption in the derivation of the PSE. ${ }^{20}$ The findings of Sandham and Salgado and Cheung et al., regardless of the relatively low Reynolds number, are however important as there is experimental evidence $^{22}$ that instability waves are present in fully turbulent jets at high Reynolds number. In the present study we therefore investigate inflow conditions based on linearly unstable eigenmodes and attempt to clarify effects of the inflow forcing amplitude on the transitional behavior of the jet. From changes in transition and resulting variations of the aerodynamic properties we aim at inferring the dominant modes and structures in the breakdown process of the jet. Possibly, in the combination with the directly computed acoustic near-field, conclusions might be drawn about the underlying mechanisms of noise generation focusing, in particular, on the early nonlinear mode interactions.

This paper is organized as follows. In Sec. II the numerical code and physical parameters of the jet flow are described. The results, given in Sec. III, are subdivided into instantaneous data in Sec. III A, mean flow results in Sec. III B followed by results for the directly computed jet noise in Sec. III C Finally, in Sec. III D, an attempt is made to establish a link between the tonal component present in the acoustic near-field and the events in the flow field. A summary and conclusions are given in Sec. IV.

\section{SIMULATION APPROACH}

\section{A. Governing equations and numerical method}

Our simulation code is based on a conservative formulation of the compressible Navier-Stokes equations expressed in generalized coordinates. ${ }^{23}$ We employ a mapping from Cartesian $(x, y, z)$ to cylindrical $(r, \theta, z)$ coordinates to retain the conservative formulation which eliminates problems related to the specific numerical treatment of additional force terms (centrifugal force and Coriolis force) that arise in other formulations. The singularity of the governing equations in cylindrical coordinates at $r=0$ is treated by an approach which uses a shifted grid in the radial direction and thus, avoids placing a grid point at the pole. ${ }^{24}$ By doing so, no change in the numerical scheme in the region of interest becomes necessary and differentiation can be performed across the centerline without need for any boundary scheme. In the azimuthal direction $(\theta)$ a Fourier spectral method is employed. As a result of the cylindrical coordinate system the azimuthal grid spacing becomes excessively fine near the pole. To avoid unnecessarily small time steps, the number of retained Fourier modes is linearly reduced toward the pole. ${ }^{25}$ For the LES presented herein, the convective as well as the diffusive terms are discretized in the radial $(r)$ and axial $(z)$ directions using a tenth-order compact central scheme ${ }^{26}$ at interior points. Toward the streamwise and radial boundaries the order is gradually reduced to sixth, fifth, and third order, where the latter two stencils are asymmetric. The approximation properties of the first derivative can be found in the work of Lele. ${ }^{26}$ Note that for the tenth-order scheme employed in the interior the modified wavenumber $\widetilde{\omega}$ is close to the exact wavenumber for $\omega \leq 3 \pi / 4$.

The grid is stretched in the radial and axial directions to adequately resolve the jet shear layers and the downstream development. In order to satisfy the symmetry constraint at the pole, the radial grid stretching is based on a family of mappings originally proposed to enhance the accuracy of pseudospectral approximations. ${ }^{27}$ Time integration uses a recently introduced low-storage explicit fourth-order accurate Runge-Kutta method optimized with respect to its dispersion and dissipation properties. ${ }^{28}$

For the LES, the filtered set of the governing equations is solved which leads to unclosed SGS terms. As SGS model we use the ADM-RT (relaxation term) model for compressible flows. ${ }^{29,30}$ The relaxation term is added to the right-hand side of the governing equations and acts only on the band of smallest resolved scales. Following the nomenclature of Stolz et al. ${ }^{29}$ the deconvolution operator $Q_{N}$ applied to the primary filter $G$ describes the form of this explicit secondary filter. In Fig. 1 the transfer functions of the primary and secondary filter, $\hat{G}$ and $\hat{Q}_{N} \cdot \hat{G}$, are shown along with the transfer function of the approximate inverse $\hat{Q}_{N}$ for the de- 


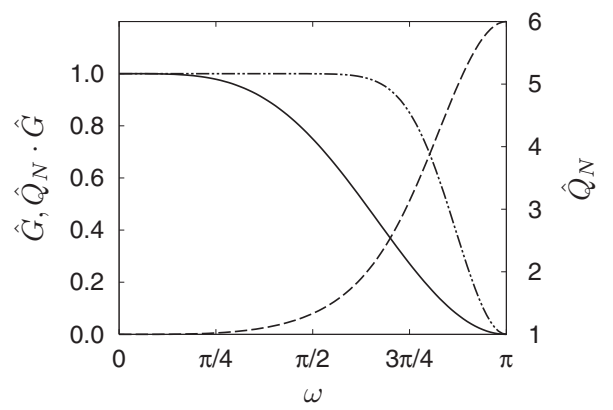

FIG. 1. Transfer functions of (-) explicit primary filter $\hat{G},(---)$ approximate inverse $\hat{Q}_{N}$, and $(-\cdot-)$ secondary filter $\hat{Q}_{N} \cdot \hat{G}$ for $N=5$.

convolution order $N=5$ employed throughout this study. Previous work ${ }^{30,31}$ has demonstrated excellent results with this approach. The nondimensional governing equations solved here are thus given by

$$
\begin{gathered}
\frac{\partial\left(J^{-1} \mathbf{Q}\right)}{\partial t}+\frac{\partial\left[J^{-1}\left(\mathbf{F}_{\mathbf{E}}-\mathbf{F}_{\mathbf{S}}\right)\right]}{\partial \xi}+\frac{\partial\left[J^{-1}\left(\mathbf{G}_{\mathbf{E}}-\mathbf{G}_{\mathbf{S}}\right)\right]}{\partial \eta} \\
+\frac{\partial\left[J^{-1}\left(\mathbf{H}_{\mathbf{E}}-\mathbf{H}_{\mathbf{S}}\right)\right]}{\partial \zeta}=-\chi\left(I-Q_{N} * G\right) * \mathbf{Q},
\end{gathered}
$$

where $\mathbf{Q}$ denotes the vector of conservative variables $\mathbf{Q}=[\rho, \rho u, \rho v, \rho w, E]^{T}$ and $J$ is the Jacobian of the coordinate transformation. $\mathbf{F}_{\mathbf{E}}, \mathbf{G}_{\mathbf{E}}$, and $\mathbf{H}_{\mathbf{E}}$ are the convective fluxes whereas $\mathbf{F}_{\mathbf{S}}, \mathbf{G}_{\mathbf{S}}$, and $\mathbf{H}_{\mathbf{S}}$ are the viscous fluxes. ${ }^{32}$ In Eq. (1) the variable $t$ denotes time, and $\xi, \eta$, and $\zeta$ are the equidistant computational coordinates. The equation of state for a perfect gas is assumed to be valid and the viscosity is determined using Sutherland's law. The relaxation coefficient $\chi$ is set equal to the inverse of the computational time step, $\chi=1 / \Delta t$, in this study.

A sketch of the computational domain and the employed boundary conditions is shown in Fig. 2. At all boundaries of the computational domain, nonreflecting boundary conditions $^{33}$ are implemented where, in addition, the curvilinear formulation at the lateral boundary is taken into account. $^{34}$ These boundary conditions are supplemented by sponge layers ${ }^{35,36}$ to define the inflow condition and the lateral and outflow boundary conditions. In the inflow sponge region, disturbances equivalent to amplified eigenmodes predicted by linear stability theory (LST) are superimposed on a hyperbolic-tangent inflow profile in a similar fashion as done by Lui and Lele ${ }^{37}$ and Bodony and Lele. ${ }^{7}$ For an example of

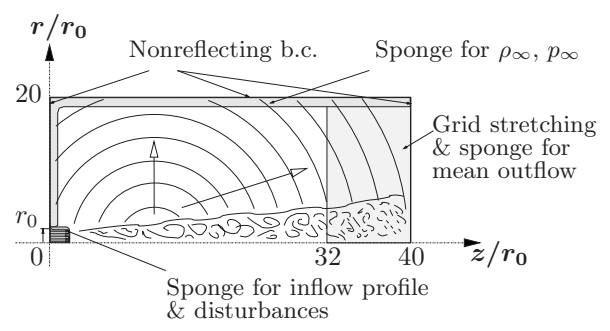

FIG. 2. Sketch of computational domain, boundary conditions, and simulation setup. Shaded areas denote sponge layers and the grid-stretching area/ sponge layer in the outflow region. instability-wave excitation in jet noise investigations we refer to the works of Bodony and co-worker, ${ }^{8,36}$ where, in particular, the latter addresses issues of the sponge technique in this context. Implementation details of the disturbance triggering can be found in Appendix A. Close to the jet-inflow region a sponge surrounding the computational domain in the radial and upstream directions is employed to prevent a mean pressure drift reported for example in the work of Colonius et $a l .{ }^{3}$ This small sponge region exclusively acts on density and pressure and maintains their ambient-state values, eliminating the need to impose any reference solution there such as results of a precursor RANS simulation as done in Refs. 7 and 8. In the following, we refer to this sponge region as the ambient sponge described in more detail in Appendix B.

At the outflow the instantaneous flow field is relaxed toward a spatially varying axisymmetric mean flow to prevent spurious acoustic reflections generated by passing vortices that violate the $1 D$ nonreflecting boundary conditions. This outflow sponge reference solution is based on a threedimensional simulation of the same setup without an outflow sponge averaged over 25 flow-through times $L_{z} / U_{j}$ as well as in the azimuthal direction. The same outflow reference solution is employed for all simulations. Further details can also be found in Appendix B.

\section{B. Specification of jet flow and simulation parameters 1. Jet flow parameters}

The subject of this study is an isothermal round jet, i.e., the jet-to-ambient temperature ratio is $T_{j} / T_{\infty}=1$. The jet Mach number $\mathrm{Ma}=U_{j} / \sqrt{\gamma R T_{j}}$ is $\mathrm{Ma}=0.9$ using $\gamma=1.4$ and common notation. The governing equations are nondimensionalized using the jet radius $r_{0}=D_{j} / 2$, velocity $U_{j}$, jet density $\rho_{j}$, the dynamic viscosity $\mu_{j}$, and jet temperature $T_{j}$ (all taken at the nozzle exit on the jet centerline). The Reynolds number $\operatorname{Re}=\rho_{j} U_{j} D_{j} / \mu_{j}=4.5 \times 10^{5}$ is between those of the reference cases at $\mathrm{Re}=1.0 \times 10^{6}$ and $\mathrm{Re}=5.0 \times 10^{5}$ (experiments by Lau et al. ${ }^{38}$ and Arakeri et al., ${ }^{39}$ respectively) and at $\mathrm{Re}=4.0 \times 10^{5}$ (LES by Bogey and Bailly ${ }^{6,16}$ ). The differences in the viscous effects associated with the different Reynolds number are here considered negligible. ${ }^{40}$

\section{Computational grid and simulation parameters}

The cylindrical computational domain with dimensions $L_{r} / r_{0}=20$ and $L_{z} / r_{0}=40$ is discretized using $N_{r} \times N_{\theta} \times N_{z}$ $=237 \times 50 \times 349\left(\approx 4.1 \times 10^{6}\right)$ grid points. A cut through the computational grid in two planes is shown in Fig. 3. In the radial direction the grid is refined in the vicinity of the shear layers and has a minimum radial spacing of $\Delta r / r_{0}=0.05$ at $r / r_{0}=1$. It is stretched toward the boundary with a maximum spacing of $\Delta r / r_{0}=0.28$ at the radial boundary. In the streamwise direction, the grid spacing is of constant size $\Delta z / r_{0}=0.1$ up to $L_{z} / r_{0} \approx 30$ and continuously increased beyond. The maximum grid spacing $\Delta z / r_{0}=0.6$ is reached at the outflow boundary $L_{z} / r_{0}=40$. Only a small fraction of the radial domain is covered by the ambient sponge, and because of the low sponge amplitude the physical region of the domain covers $L_{r} / r_{0}=19.4$ and $L_{z} / r_{0}=32$. 
(a)

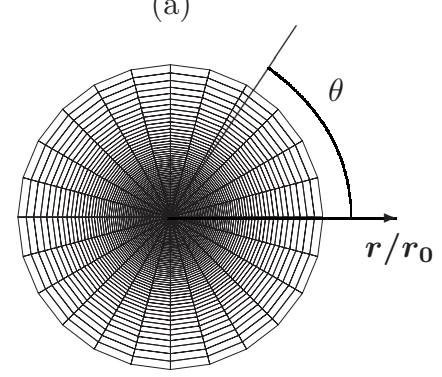

(b)

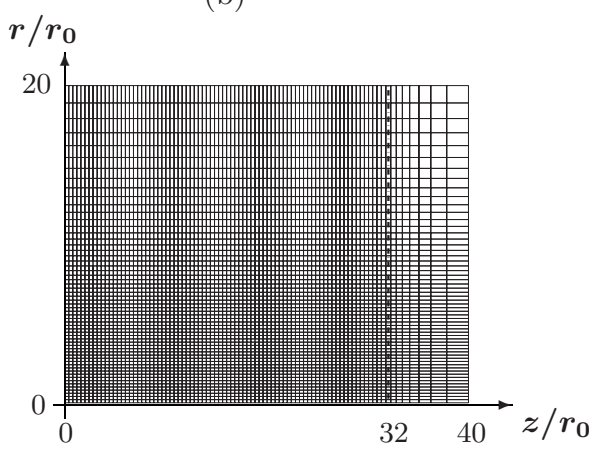

FIG. 3. Computational grid in (a) the $r-\theta$ plane and (b) the $r-z$ half plane. In the azimuthal direction only every second grid line is shown of a grid corresponding to $N_{\theta}=48$ points. Only every fourth grid line is shown in the radial and axial directions. The vertical line $(-\cdot-)$ denotes the beginning of the outflow sponge zone.

The resolution of the grid in combination with the employed discretization schemes determines the maximum frequency that can be represented. This frequency, sometimes referred to as grid cutoff frequency or in dimensionless terms as grid Strouhal number $\mathrm{St}^{g}$ (Ref. 8) can be determined approximately for waves that are perfectly aligned with the grid. In the part of the computational domain where acoustic nearfield data will be provided, the grid cutoff is $\mathrm{St}^{g} \approx 5$ at the downstream observer location for downstream propagating waves and, due to the radially stretched grid, $\mathrm{St}^{g} \approx 3$ for the sideline observer location for radially propagating waves. Note that the effective cutoff depends on the phase speed anisotropy and on the propagation direction due to the grid anisotropy.

The simulation time for all cases presented hereafter is $T_{\text {sim }}=1000 r_{0} / U_{j}$ which corresponds to a minimal Strouhal number of $\mathrm{St}=D_{j} /\left(T_{\mathrm{sim}} U_{j}\right)=2 \times 10^{-3}$. The time step is set to $\Delta t=0.02 r_{0} / U_{j}$, where every fifth time step is analyzed for the flow statistics (10 000 samples) and every tenth step is stored on disk for the acoustic analysis and postprocessing (5000 samples). Using the Nyquist theorem these sampling rates correspond to maximum resolved Strouhal numbers of $\mathrm{St}_{N}=10$ for the flow data and $\mathrm{St}_{N}=5$ for the velocity spectra, azimuthal correlation coefficients and near-field spectra of the pressure. To reduce the variance of the spectra, the sampling interval is divided into subintervals that overlap by $50 \%$ and all data in the azimuthal direction is employed, i.e., the spectra are averaged over the number of subintervals and azimuthal grid points $N_{\theta}$. The side effects of employing finite-time intervals are reduced by applying a Hann window to each subinterval before performing a discrete Fourier transform in time. The interval length for the analysis of the velocity spectra is selected such as to resolve a nondimensional frequency of $\mathrm{St}=0.04$. Similar to Ref. 41 the pressure signals are analyzed using shorter subintervals that resolve a minimal Strouhal number of $\mathrm{St}=0.1$. In order to determine the frequency of a tonal contribution observed in the pressure spectra more accurately, an analysis with increased interval length has been performed in addition.

\section{Inflow disturbance seeding}

At the inflow a hyperbolic-tangent base-flow profile representative for jet flow profiles close to the jet nozzle exit, ${ }^{42}$

$$
\frac{w_{b}}{U_{j}}=\frac{1}{2}\left\{1+\tanh \left[\frac{r_{0}}{2 \theta_{0}}\left(1-\frac{r}{r_{0}}\right)\right]\right\},
$$

is imposed where the initial momentum thickness is set to $\theta_{0} / r_{0}=1 / 20$. This value is chosen such that the steep gradient of the velocity profile can adequately be resolved by the grid used and thus, in numerical studies and in LES, in particular, is driven by computational cost constraints. Experimentally observed initial momentum thicknesses can be smaller by at least an order of magnitude. Zaman,${ }^{14}$ for example, reports $\theta_{0} / r_{0} \approx 1 / 200$ for jets at Reynolds numbers of $\operatorname{Re}_{D} \gtrsim 2.5 \times 10^{5}$. Correspondingly, a jet exit boundary layer with the currently investigated initial momentum thickness would be laminar and at Reynolds numbers below $10^{5}$, as also supported by the data of Zaman.

In order to trigger transition to turbulence, a number of instability modes of the inflow profile are superimposed onto the base flow. To determine these, a classical wave ansatz for the disturbances is introduced into the linearized compressible Navier-Stokes equations in cylindrical coordinates and the resulting eigenvalues problem is solved using a Chebyshev collocation method. ${ }^{43,44}$ Different azimuthal wavenumbers $n$ are investigated with respect to their linear spatial stability properties. Note that at the inflow plane two length scales are appropriate for the jet flow, the initial momentum thickness $\theta_{0}$ and the jet diameter $D_{j}$. Here, we rely on a scaling of the frequency $\omega$ based on the diameter $D_{j}$ because we are interested in the downstream development where the thickness becomes comparable to $D_{j}$ and thus curvature effects become important. The growth rates as well as phase speeds associated with the viscous instabilities of the inflow profile [Eq. (2)] are shown in Fig. 4 as a function of the Strouhal number $\mathrm{St}=\omega D_{j} /\left(2 \pi U_{j}\right)$. In Fig. 4(a), the growth rate is indicated by the imaginary part of the streamwise wavenumber $\alpha_{i}$ and in Fig. 4(b) the phase speed of the disturbances $\omega / \alpha_{r}$ is shown. In agreement with results reported in literature ${ }^{42}$ we find the axisymmetric or varicose mode $n=0$ to be least stable reaching maximum amplification around $\mathrm{St}=0.676$. This corresponds to $\mathrm{St}_{\theta}=\omega \theta_{0} /\left(2 \pi U_{j}\right)=0.0169$ which is in good agreement with the inviscid theoretical value of $\mathrm{St}_{\theta}=0.0165 .{ }^{42}$ At low frequencies $\omega$ the growth rates $-\alpha_{i}$ for mode $n=0$ are no longer dominant and for $\mathrm{St} \leq 0.42$ the helical mode $n=1$ is least stable. In Fig. 4(b) one finds that all disturbances with St $>0.8$ propagate with similar phase speed $\omega / \alpha_{r}$. 
(a)

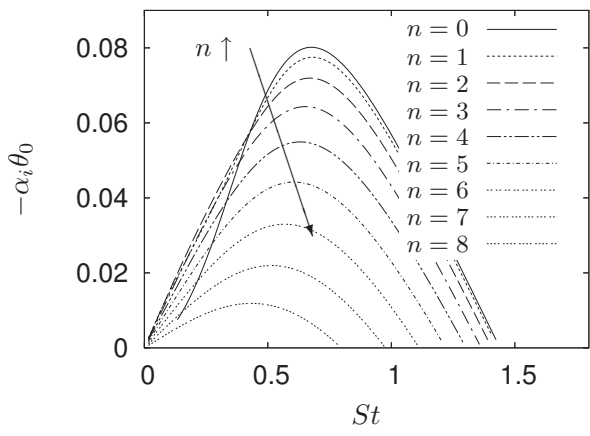

(b)

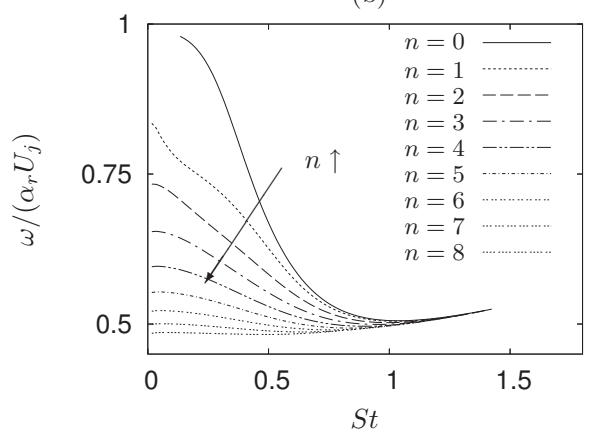

FIG. 4. (a) Growth rate $-\alpha_{i}$ vs St and (b) phase speed $\omega / \alpha_{r}$ vs St for different azimuthal wavenumbers $n$.

In the present study we exclude the low-order modes $n \leq 3$ from the forcing for two reasons: First, Bodony and Lele ${ }^{12,45}$ reported overestimated sound pressure levels (SPLs) and link it to the dominant axisymmetric mode when disturbing LST modes $n=0, \pm 1, \pm 2$ in their study. Second, Bogey and Bailly ${ }^{16}$ reported a delayed streamwise development of the jet flow and observe a reduction of SPL when excluding modes $n=0$ through $n=3$ from their ring-vortex forcing method. Note that the numerical reference data ${ }^{16}$ employs a total of sixteen azimuthal modes $|n| \leq 15$ for the inflow excitation. Here, the inflow disturbances are seeded by superimposing a total of ten eigenmodes onto the base flow. Left- and right-turning azimuthal wavenumbers $|n|= \pm n=4, \ldots, 8$ are excited at the frequencies which give the maximum growth rate. In the following we refer to these frequencies as the fundamental ones. The wavenumbers and corresponding forcing frequencies are $(|n|, \mathrm{St})=(4,0.63)$, $(5,0.61),(6,0.57),(7,0.51)$, and $(8,0.43)$. The linear superposition of the eigenmodes is given by

$$
\begin{aligned}
\mathbf{Q}_{\text {infl }}(r, \theta, z, t)= & \mathbf{Q}_{\mathrm{b}}(r)+\sum_{|n|=4}^{8} \mathbf{Q}_{n}^{\prime}(r, \theta, z, t) \\
= & \mathbf{Q}_{\mathrm{b}}(r)+A_{\text {total,LST }} \\
& \times \frac{\sum_{|n|=4}^{8} A_{n} \Re\left[\hat{\mathbf{Q}}_{n}(r) e^{i\left(\alpha_{n} z+n \theta-\omega_{n} t+\phi_{n}\right)}\right]}{\sum_{|n|=4}^{8} A_{n}},
\end{aligned}
$$

where $\mathbf{Q}_{\text {infl }}$ denotes the conservative variables $\mathbf{Q}$ at the inflow. In Eq. (3), $\mathbf{Q}_{\mathrm{b}}$ denotes the base flow and $\hat{\mathbf{Q}}$ the complex eigenfunction that is normalized by setting $\max |\hat{w}|=1$. The laminar base-flow profile $\mathbf{Q}_{\mathrm{b}}(r)$ is derived from Eq. (2), em-
TABLE I. Nomenclature and inflow forcing parameters of cases investigated.

\begin{tabular}{lcc}
\hline \hline Case & $A_{\text {total,LST }}$ & Modes $|n|$ \\
\hline Low & 0.015 & $4, \ldots, 8$ \\
Med & 0.030 & $4, \ldots, 8$ \\
High & 0.045 & $4, \ldots, 8$ \\
\hline \hline
\end{tabular}

ploying the Crocco-Busemann relation for an isothermal jet flow as well as the linearized equation of state. The eigenmodes are modulated in amplitude $A_{n}$ and phase $\phi_{n}$ in a random walk fashion (see Appendix A for details) in order to prevent phase locking. ${ }^{7}$ The superposition of instability waves is scaled with the sum of amplitudes $A_{n}$ to obtain the total disturbance amplitude $A_{\text {total,LST }}$ which will be varied parametrically. The disturbed inflow profile $\mathbf{Q}_{\text {infl }}$ is imposed by the inflow sponge in order to prevent reflections at the inflow as well as to allow for entrainment of surrounding fluid toward the jet shear layers. The reference state is defined by Eq. (3), i.e., by a spatially and temporally varying superposition of linearly unstable modes onto a laminar velocity profile.

In this study, we restrict ourselves to unstable modes (according to the viscous linear stability theory these are azimuthal wavenumbers $n=0, \ldots, 8)$ and focus on the influence of changes of disturbance amplitude $A_{\text {total,LST }}$ on the flow field development and its associated noise. This ensures consistency with the complete set of governing equations in contrast to, e.g., using the ring-vortex disturbance method. ${ }^{9,16}$ The effect of a change in $A_{\text {total,LST }}$ is investigated in detail to further clarify the sensitivity of transitional jet simulation results to inflow disturbances. The variation of the forcing amplitude is expected to alter the noise generation mechanism and, hence, might offer the possibility to investigate the relative contributions of different sources. Using linear stability disturbances the effect of the nonlinear development of eigenmodes and their role in the transition process and the resulting noise can be assessed. In the following three cases are reported with parameters given in Table I: cases Low, Med, and High with a total disturbance amplitude of $A_{\text {total,LST }}=1.5 \%, 3.0 \%$, and $4.5 \%$ based on the jet exit velocity.

\section{RESULTS}

\section{A. Instantaneous data}

Figure 5 shows snapshots of the vorticity magnitude $|\boldsymbol{\omega}|$ for the three investigated cases in the developing region of the shear layers. At first sight, the jet shear layer development looks similar for all cases. However, the specific details of the transitional behavior are different: with increasing disturbance amplitude the rollup process is shifted in the upstream direction. As the shear layers roll up (marked by (1) in all graphs), we observe large dominant structures for case Low. In contrast, when enforcing larger disturbance amplitudes, as done in cases Med and High, the developing shear layer roll up into smaller vortical structures. For lowamplitude forcing consecutive structures are spatially sepa- 
(a)

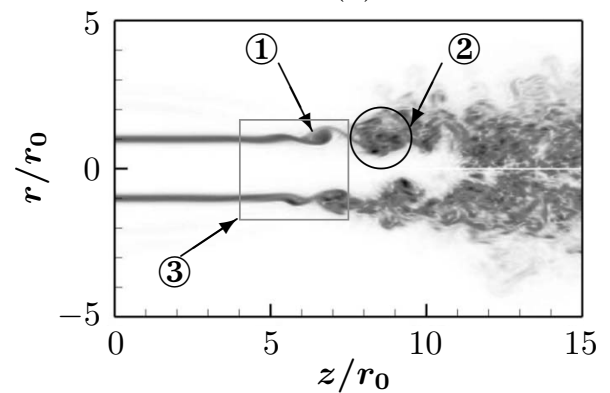

(b)

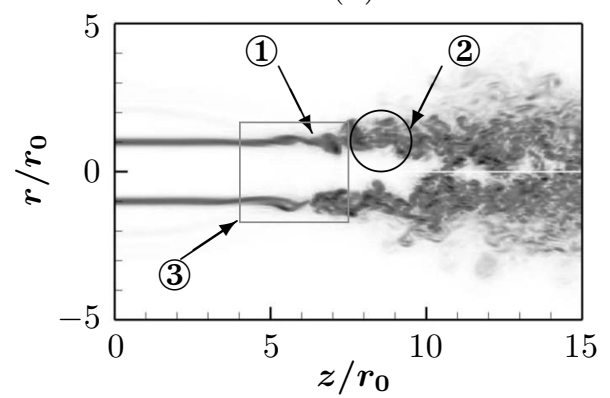

(c)

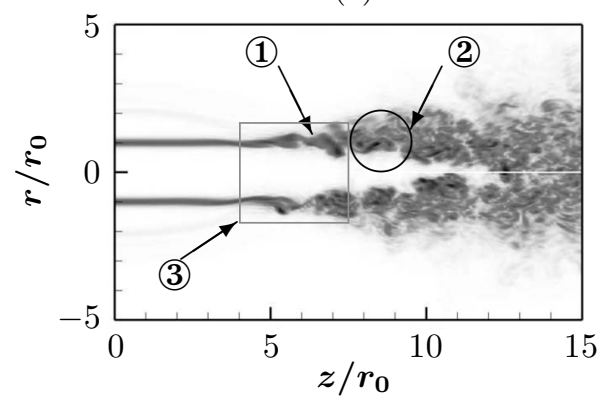

FIG. 5. Visualization of vortical structures by $|\boldsymbol{\omega}|$ in the $r-z$ plane at $\theta=0$ for cases (a) Low [URL: http://dx.doi.org/10.1063/1.3112686.1], (b) Med [URL: http://dx.doi.org/10.1063/1.3112686.2], and (c) High [URL: http:// dx.doi.org/10.1063/1.3112686.3]. The gray contour levels from light to dark are within $0 \leq|\boldsymbol{\omega}| \leq 13$. (1) dominant structure during rollup of shear layer; (2) interaction of large-scale structures; (3) gray frame is the zoomed region of Fig. 6 (enhanced online).

rated and start to interact downstream of $z / r_{0} \approx 7$, whereas for larger disturbance amplitude the structures merge and interact more quickly. As these structures are convected downstream, the breakdown process continues and smaller and smaller scales are generated leading to several vortex cores marked by a high concentration of vorticity (see encircled area (2)). Clearly, the spatial extent in which the breakdown into smaller scales takes place is much shorter for case Low compared to a more moderate and spatially elongated process for the higher amplitude cases. The gray box in each graph (3) shows the extent of the transitional region to be looked at in more detail in Fig. 6.

The flow animations (see Fig. 5 enhanced online) show vortex pairings of the large-scale structures. Compared to a tonally excited jet flow or mixing layer these pairings are less pronounced and occur only occasionally. The occurrence is disguised by the random rollup process which introduces a variation of the spatial location as well as a jitter of the scales. To some extent, this randomness is reduced by increasing the forcing amplitude, as we can see from the ordering of the vortices: they are ordered in a helical manner at opposite sides of the shear layer for case Low, whereas the mechanism for case High seems to be dominated by an axisymmetric component.

In Fig. 6, the transition regions of the three jets are visualized in three different views using the $\lambda_{2}$ vortex identification criterion ${ }^{46}$ for the same vortical structures as before. The quality of the isosurface visualizations is enhanced by spectrally interpolating the data onto $N_{\theta}=300$ allowing a more accurate representation of isosurfaces. The isosurfaces $\lambda_{2}=-1$ show the same upstream-shifted transition as in the previous visualizations of the vorticity magnitude. However, using the $\lambda_{2}$ criterion the differences in spatial extent of the structures become more evident, as can be seen in the side view given in row (A). For case Low, large azimuthally correlated structures dominate the rollup whereas threedimensional and streamwise-elongated small vortex filaments or vortex tubes initiate the breakdown process for higher disturbance levels. The front views in row (B) show that the hairpin vortices emerging in the radial direction have significantly smaller azimuthal spacing but increased radial extent for larger forcing amplitude. In the three-dimensional view presented in row (C) the strong azimuthal coherence and the interaction of the structures for case Low can be seen, whereas cases Med and High show significantly distorted toroidal structures which disintegrate into small structures. In summary, the transitional development is initiated at larger distances from the inflow and is more rapid when using the low excitation level. Thereby, larger, clearly separated structures are generated in the shear layer. However, the position of interacting structures itself is localized with increasing forcing amplitude and occurs in a more axisymmetric fashion. To support these observations quantitatively, the one-dimensional azimuthal turbulent kinetic energy spectra will be investigated in Sec. III B.

To obtain an impression of the instantaneous near-field noise of the jet flow, the same data is visualized again in Fig. 7 , together with the pressure fluctuations $p^{\prime}$ in the outer part of the domain. We only present case Med since the differences in the visualizations of the instantaneous near-field pressure data are minute for the investigated cases. For all simulated cases, sound waves are emitted slightly upstream of the position where the instantaneous shear layers merge. At the radial and upstream boundaries, all pressure waves propagate outwards without causing any reflections. This demonstrates that the chosen combination of nonreflecting boundary conditions supplemented by sponge layers is appropriate.

\section{B. Mean flow and turbulence results}

The influence of different forcing amplitudes on the mean flow and jet development is now investigated. The mean flow is visualized in Fig. 8 to assess the quality of the current simulation setup to model free-stream boundaries and to allow for entrainment of surrounding fluid. Unless stated otherwise, we denote mean quantities by $\langle\cdot\rangle$, determined by 
(a)

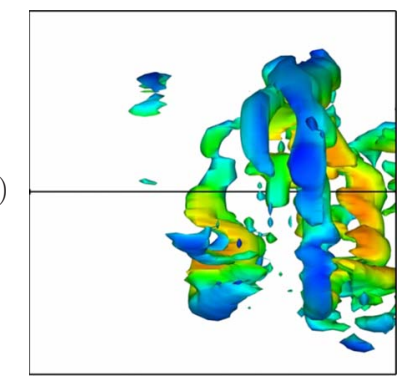

(B)

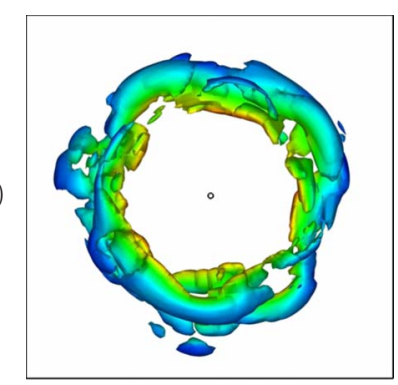

(C)

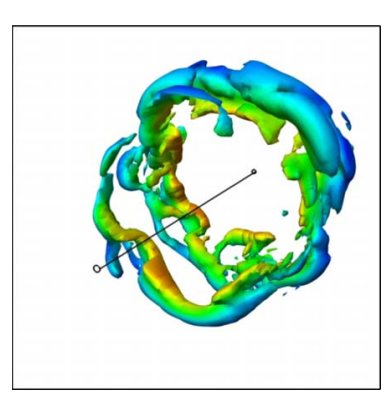

(b)
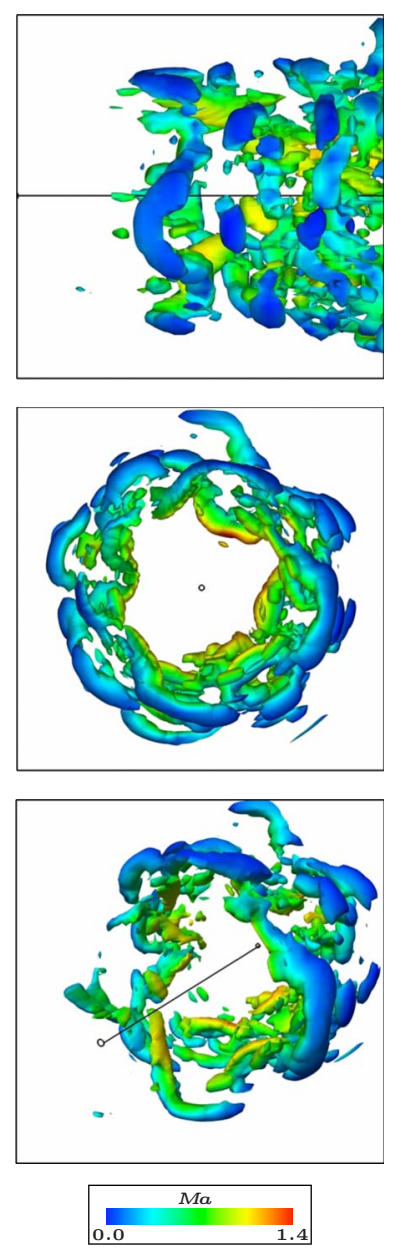

(c)
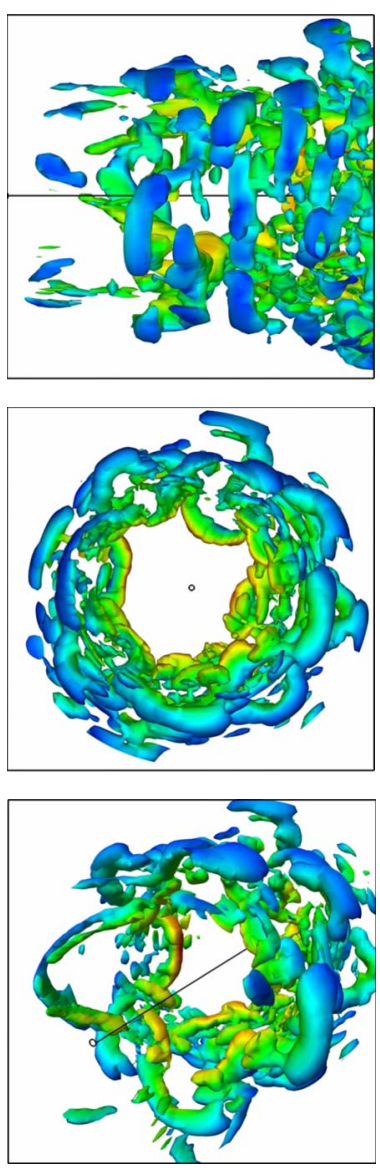

FIG. 6. (Color online) Visualization of vortical structures by isosurfaces of the $\lambda_{2}$ vortex identification criterion (Ref. 46) in regions $-1.725 \leq r / r_{0}$ $\leq 1.725$ and $4 \leq z / r_{0} \leq 7.5\left(\lambda_{2}=-1\right)$. (a) Low, (b) Med, and (c) High in a (A) side view, (B) front view, and (C) three-dimensional front-side view. The dimensions of the shown graphs in row (A) correspond to the gray frame in Fig. 5. The isosurface is (color) coded by the local Mach number 0 $\leq \mathrm{Ma} \leq 1.4$ from dark gray (blue) to light gray (red). averaging over time and in azimuthal direction. The length of the potential core $z_{c}$, defined by the position where the mean axial velocity on the centerline $w_{c}=\langle w(r \approx 0)\rangle$ is equal to $0.95 U_{j}$, is $z_{c} / r_{0} \approx 12.17$. The dotted-dashed line corresponds to the mean flow value $\langle w\rangle=0.95 U_{j}$, whereas the dashed line denotes $\langle w\rangle=0.05 U_{j}$ and exhibits a rather linear spreading of the jet downstream of around five jet diameters. The solid line marks half the jet exit velocity and demonstrates the slow decay of axial momentum which necessitates large computational domains. The streamline patterns of the other excitation levels differ only slightly and are therefore not shown. The potential core lengths $z_{c}$ vary slightly with forcing amplitude, and are listed in Table II. With an increased forcing amplitude, we find reduced potential core lengths as expected. In the same table other data characterizing the jet development can be found, such as the growth rate of the jet half-width, the virtual origin and the growth rate of the vorticity thickness.

The streamwise development of the mean axial velocity at the centerline $w_{c}$ and the jet half-width $r_{1 / 2}$, given by $\left\langle w\left(r=r_{1 / 2}\right)\right\rangle=0.5 w_{c}$, are shown in Fig. 9. Since the forcing is of Kelvin-Helmholtz instability type one can expect differences along the jet centerline to remain small upstream of the potential core collapse. With increased forcing amplitude, the centerline velocity starts to decay slightly further upstream, while the decay itself remains almost unaffected. The results compare favorably with the experimental reference data by Lau et al. ${ }^{38}$ and Arakeri et al. ${ }^{39}$ as well as with the numerical data by Bogey and Bailly. ${ }^{16}$ The reference LES has been shifted in the axial direction by $z_{0} / r_{0}=2$ in all graphs to match the different core lengths. The experimental data denoted by symbols is shifted as follows: that of Arakeri et al. by $z_{0} / r_{0}=-5$ and that of Lau et al. by $z_{0} / r_{0}=-4$. The numerical results exhibit a streamwise delayed but a slightly more abrupt onset of velocity decay. In the experiments of Lau et al. a faster decay of the mean centerline velocity is observed leading to the lowest mean flow velocities in the investigated domain. Note that without this axial shift the experimental data exhibit a delayed potential core collapse compared to the numerical data. This delayed but more prompt onset of decay for the computational results might be related to the aforementioned differences in the initial momentum thicknesses and in oncoming disturbance levels and has been similarly reported in other investigations. ${ }^{7,12,16}$ The disturbance-level effect, although small, is best seen for case Low, where the delayed onset combined with an enhanced decay eventually causes the distribution to cut across the medium-amplitude one.

The axial development of the jet half-width shown in Fig. 9(b) varies only little for the range of investigated inflow forcing levels. At the downstream position $z / r_{0} \approx 5$ the jet spreading sets in. Downstream of the potential core the 
(a)

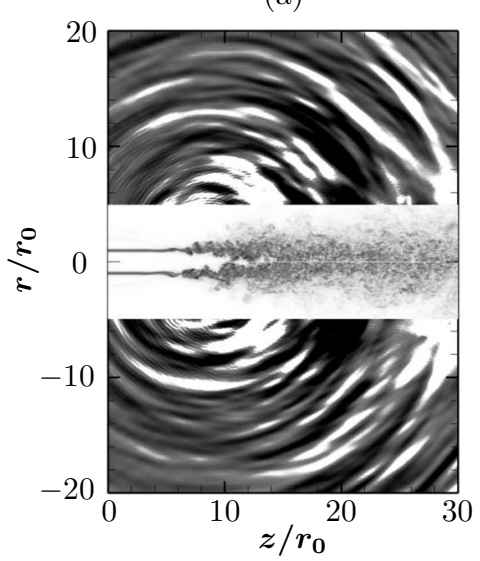

(b)

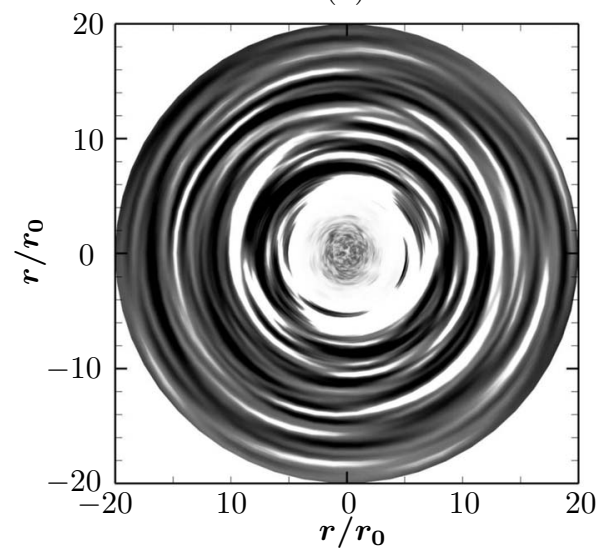

FIG. 7. Visualization of vortical structures by contours of $|\boldsymbol{\omega}|$ in the nearfield $r / r_{0}<5$ (gray contour levels from light to dark within $0 \leq|\boldsymbol{\omega}| \leq 13$ ) and corresponding pressure fluctuations in the acoustic near-field $r / r_{0} \geq 5$ (gray contour levels from dark to light within $-5 \times 10^{-4} \leq p^{\prime} \leq 5 \times 10^{-4}$ ) for case Med: (a) in the $r-z$ plane at $\theta=0$ and (b) in the $r-\theta$ plane at $z / r_{0}=12$.

spreading is significantly increased and the jet widens almost linearly in the streamwise direction. Since the streamwise extent of the computational domain is too short to contain large parts of the self-similar region the spreading rates of the jet flow have not been determined. Nevertheless, the growth rates of the jet half-width for all simulations (determined by a linear fit to the data within $15 \leq z / r_{0} \leq 30$ ) are given in Table II. They are between $d\left(r_{1 / 2} / r_{0}\right) / d z \approx 0.052$ and 0.062 as similarly reported by Bogey and Bailly, ${ }^{47}$ who,

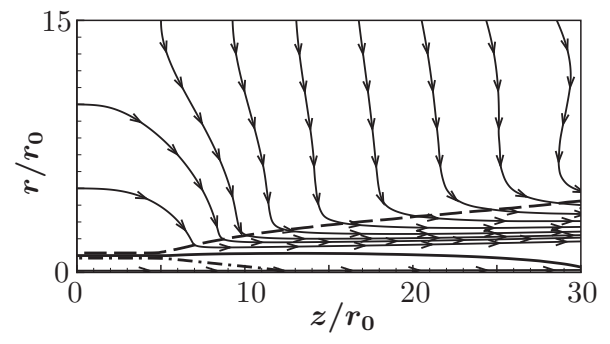

FIG. 8. Visualization of mean flow streamlines for case Med. Lines denote $(-\cdot-)\langle w\rangle=0.95 U_{j},(-)\langle w\rangle=0.5 U_{j}$, and $(---)\langle w\rangle=0.05 U_{j}$.
TABLE II. Potential core lengths $z_{c} / r_{0}$, growth rates of the jet half widths $d\left(r_{1 / 2} / r_{0}\right) / d z$ (determined by a linear fit to the data within $15 \leq z / r_{0} \leq 30$ ), virtual origins $z_{0} / r_{0}$ and growth rates of the vorticity thicknesses $d\left(\delta_{\omega} / r_{0}\right) / d z$ (determined by a linear fit to the data within $6 \leq z / r_{0} \leq 10$ ) for different simulations.

\begin{tabular}{lcccc}
\hline \hline Case & $z_{c} / r_{0}$ & $d\left(r_{1 / 2} / r_{0}\right) / d z$ & $z_{0} / r_{0}$ & $d\left(\delta_{\omega} / r_{0}\right) / d z$ \\
\hline Low & 12.63 & 0.062 & -5.23 & 0.249 \\
Med & 12.17 & 0.056 & -8.00 & 0.251 \\
High & 11.99 & 0.053 & -9.78 & 0.246 \\
\hline \hline
\end{tabular}

when simulating in a box with twice the streamwise extent, observed an increase in the downstream direction toward asymptotic values reported in literature.

The root-mean-square (RMS) of the axial and radial velocities along the jet centerline $r / r_{0}=0$ are shown in Fig. 10. Overall, the streamwise evolution of rise and subsequent fall of RMS intensities is similar for all cases. In particular, cases High and Med are almost indistinguishable. The axial fluctuations presented in Fig. 10(a) saturate close to the inflow at levels of $\left\langle w_{c}^{\prime \prime 2}\right\rangle^{1 / 2} / U_{j} \approx 0.01$. In the transition region they rise significantly until reaching their maxima around $z / r_{0}$ $=15, \ldots, 17$. With increasing forcing amplitude $A_{\text {total,LST }}$, the streamwise development is shifted slightly upstream and the corresponding peaks also occur at shorter distances from the inflow. The agreement between cases Med and High with the recent experiments of Arakeri et al. ${ }^{39}$ is very good with respect to the peak values and the streamwise evolution. Both simulations reach a RMS peak of $\left\langle w_{c}^{\prime \prime 2}\right\rangle^{1 / 2} / U_{j}=0.124$. The development of case Low is slightly delayed and reaches a

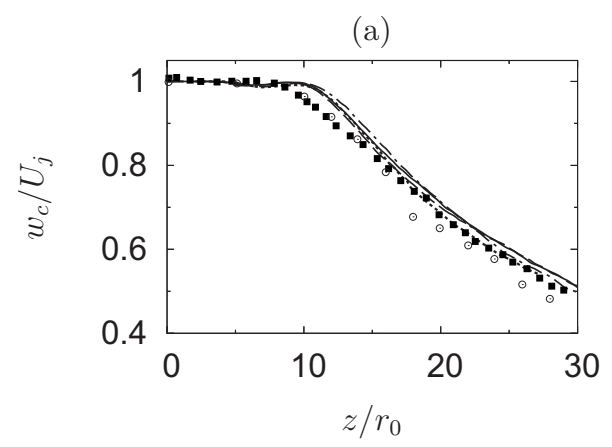

(b)

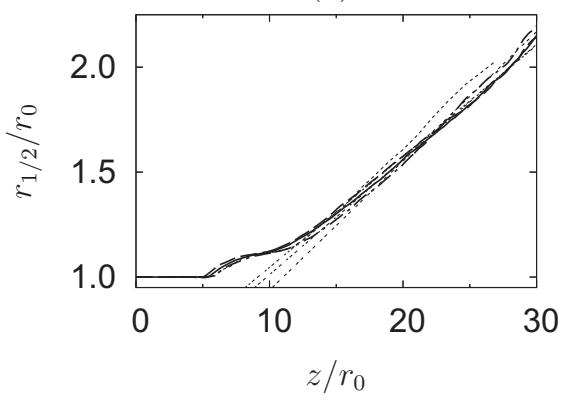

FIG. 9. (a) Mean axial velocity along the jet centerline $w_{c}$ and (b) jet half width $r_{1 / 2}$ : (-.-) Low, (-) Med, (-- ) High, ( . .) Bogey and Bailly (Ref. 16), (O) Lau et al. (Ref. 38), (ロ) Arakeri et al. (Ref. 39); (- -, - - , - . - ) denote the linear fit to the half-width development for cases Low, Med, and High. 
(a)

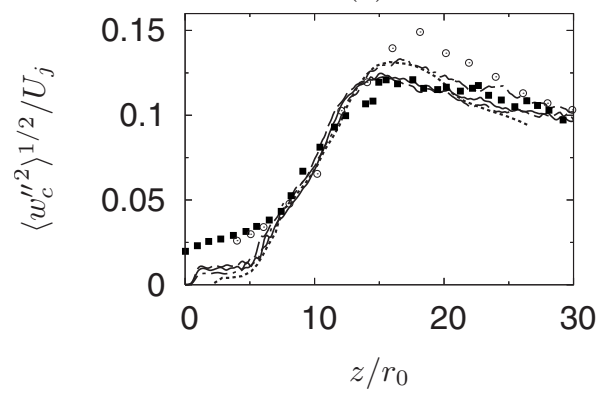

(b)

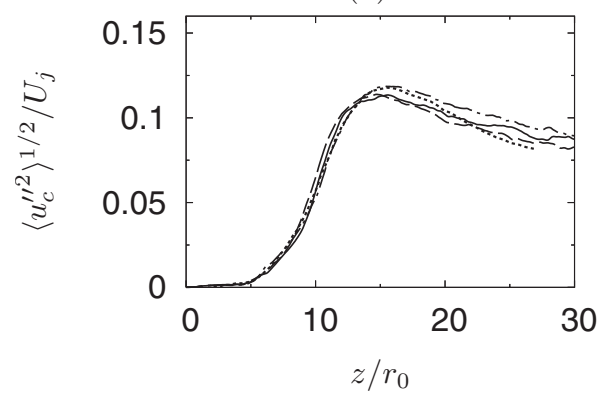

FIG. 10. RMS of (a) axial velocity fluctuations $\left\langle w_{c}^{\prime \prime 2}\right\rangle^{1 / 2}$ and (b) radial velocity fluctuations $\left\langle u_{c}^{\prime \prime 2}\right\rangle^{1 / 2}$ along the jet centerline: (-.-) Low, (-) Med, (- - ) High, (. . ) Bogey and Bailly (Ref. 16), (○) Lau et al. (Ref. 38), ( Arakeri et al. (Ref. 39).

peak value of $\left\langle w_{c}^{\prime \prime 2}\right\rangle^{1 / 2} / U_{j}=0.133$ which is in good agreement with the reference LES. ${ }^{16}$ Lau et al. ${ }^{38}$ reported the highest peak, however, this is attributed to the analysis procedure and lower values-similar to the Arakeri et al. data-were reported in Ref. 48.

Figure 10(b) shows the radial velocity RMS fluctuations together with the numerical reference data. For all forcing amplitudes very similar profiles are found. Again, case Low reaches highest peak values $\left\langle u^{\prime \prime 2}\right\rangle^{1 / 2} / U_{j}=0.120$ and its evolution up to the peak location is in very good agreement with the results by Bogey and Bailly ${ }^{16}$ despite the fact that the simulations use different numerical codes and, more importantly, different inflow forcing methods. Depending on the amplitude the LST-based forcing alters the transition and breakdown: the RMS of both velocity components along the jet centerline are increased for case Low as the result of the azimuthally coherent vortical structures described previously (see Fig. 6) that dominate the rollup of the shear layers. In contrast, lower RMS occur during the transition of cases Med and High where toroidal vortices are strongly distorted in circumferential direction indicating rising threedimensional instabilities. These differences will be addressed in more detail below.

In the following, the effect of changes in forcing amplitude on the early shear layer development is investigated. As mentioned in Sec. III A, the alterations in the transitional region are to be quantitatively supported by analyzing the one-dimensional azimuthal turbulent kinetic energy spectra. We define the spectrum at location $(r, z)$ as
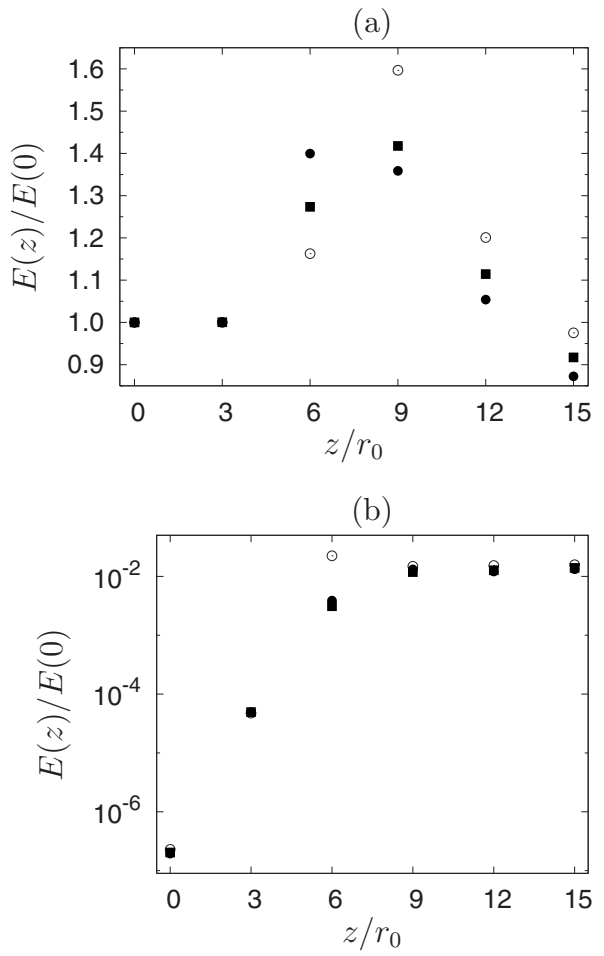

FIG. 11. One-dimensional turbulence kinetic energy of (a) varicose mode $n=0$ in linear scales and (b) first helical mode $n=1$ in logarithmic scales determined along the nozzle lip line at streamwise positions $z / r_{0}=i \times 3$ $(i=0, \ldots, 5)$ normalized by the kinetic energy of case Med at $z=0$ :

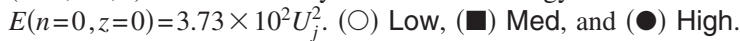

$$
E(n)=\left\langle\hat{u}_{i} \hat{u}_{i}^{*}\right\rangle,
$$

where $E(n)$ is the turbulent kinetic energy (TKE) contained in the azimuthal Fourier mode $n, \hat{u}_{i}$ denotes the onedimensional Fourier transform of the velocity component $u_{i}$ and the asterisk its complex conjugate. In Eq. (4), we use $\langle\cdot\rangle$ to denote a time average only. The one-dimensional turbulent kinetic energy of the axisymmetric mode and the first helical mode are determined at five downstream positions along the nozzle lip line $\left(r=r_{0}\right)$ and are shown in Figs. 11(a) and 11(b) for the three forcing amplitudes. The energy is normalized by the energy of case Med at the jet-inflow plane $z=0$ contained in mode $n=0$. As could be seen in the instantaneous snapshots, the vortical structures caused by instability waves alter the flow significantly at $z / r_{0} \approx 6$. At this location, enhanced TKE levels are observed and the alignment for the three cases follows their initial forcing amplitudes. The modal energy has grown by approximately $16 \%, 28 \%$, and $40 \%$ for cases Low, Med, and High, respectively. At $z / r_{0}=9$, we find that the roles are inverted: case Low exhibits the largest amount of energy, which also corresponds to the global maximum for the analyzed positions and all excitation amplitudes. Compared to the inflow level, mode $n=0$ of case Low has a $60 \%$ TKE increase. Further downstream, the differences between the three cases are reduced. However, even at $z / r_{0}=15$ the low-amplitude excitation results in higher energy in mode $n=0$ than the higher amplitude cases.

The helical mode $n=1$, which seems to dominate the instantaneous rollup process for case Low, is analyzed in Fig. 11(b). Its downstream evolution is characterized by very 


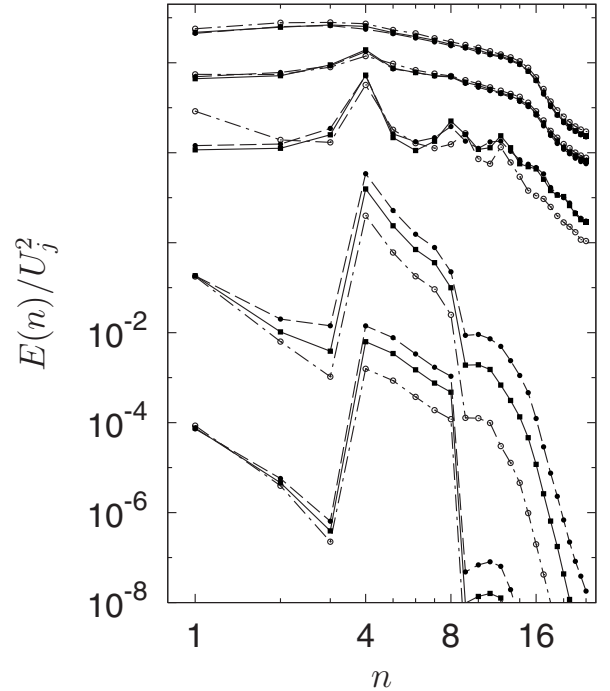

FIG. 12. One-dimensional turbulence energy spectra $E$ in the $\theta$-direction along the nozzle lip line at streamwise positions $z / r_{0}=i \times 3(i=0, \ldots, 4$ from bottom to top): (-.-) Low, (-) Med, and (- - ) High. The groups of spectra are shifted by a factor of 10 for clarity.

small initial amplitudes. A similar inversion of the alignment for the three amplitudes is observed slightly closer to the inflow region compared to $n=0$. Case Low has, for instance, a TKE increased by more than four orders of magnitude within a short streamwise distance of three nozzle diameters and exceeds the two other cases. Further downstream, this difference in mode $n=1$ for the three cases becomes gradually smaller and the results are almost indistinguishable downstream of $z / r_{0}=12$.

We now turn to the downstream development of the higher azimuthal wavenumbers, focusing in particular on the excited modes $n=4, \ldots, 8$ and the redistribution of modal energy. Five equidistant downstream locations in the transitional region along the nozzle lip line are evaluated and shown in Fig. 12. At the inflow, we see the effect of the introduced disturbances, i.e., the TKE in azimuthal wavenumbers $n=4-8$ is higher, at least by an order of magnitude compared to the unexcited modes. Also, the effect of the amplitude at the inflow is reflected by the different energy levels of the three cases. Further downstream, the energy increases in all modes, i.e., the initially undisturbed modes also rise. In particular, $n=4$ outweighs the other excited modes. The other forced modes exhibit less increase in kinetic energy and undisturbed modes, e.g., $n=1$, reach comparable values. At the third streamwise position $z / r_{0}=6$, case Low exhibits the previously noticed sudden rise of $n=1$ which substantiates the previously made observation of helical structures during its transition process. As pointed out before, this rise of mode one is accompanied by a downstream-delayed rise of TKE in the all-dominant axisymmetric mode. Significantly enhanced TKE also occurs in the even mode $n=4$ for all cases and the differences in initial amplitude are diminished. Cases Med and High are not only characterized by $n=4$ but also by higher harmonics thereof. In particular, a pronounced rise in the azimuthal wavenumber $n=8$ can be observed which seems to allow for further inter- (a)

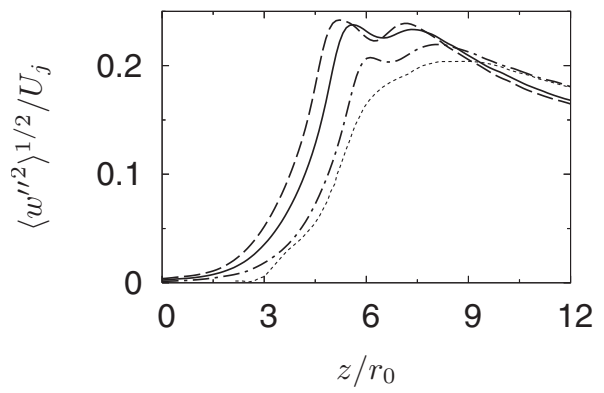

(b)

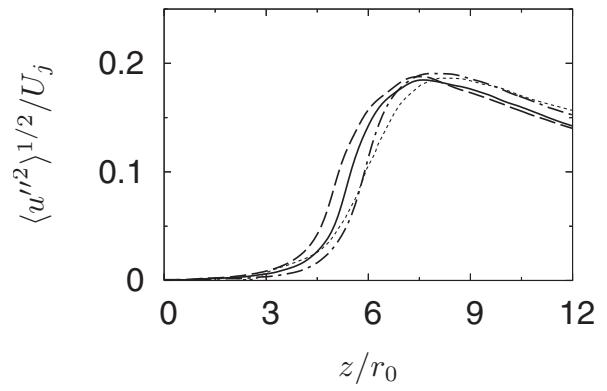

FIG. 13. RMS of (a) axial velocity fluctuations $\left\langle w^{\prime \prime 2}\right\rangle^{1 / 2}$ and (b) radial velocity fluctuations $\left\langle u^{\prime \prime 2}\right\rangle^{1 / 2}$ along the nozzle lip line: $(-\cdot-)$ Low, (-) Med, $(---)$ High, (. . ) Bogey and Bailly (Ref. 16).

actions of a weakly developed higher harmonic $n=12$. In contrast, case Low with its prominent increase in mode $n$ $=1$, exhibits only small changes in the higher wavenumbers $n=9$ and 12 and, more importantly, no distinct peak at $n=8$. A redistribution of TKE between the dominant modes might be the reason for the reduction in dominance of $n=4$ together with the rise of $n=8$, or the onset of secondary instabilities and nonlinear interactions of modes as appreciable disturbance level are attained at this downstream location. Following Hussain, ${ }^{49}$ this interaction could be related to a breakdown process of structures termed cut-and-connect, which describes the connection of two adjoining vortices. The occurrence of vortex heads in circumferential direction visualized by the $\lambda_{2}$ representation in Fig. 6 for cases Med and High compares favorably with the sketch of an idealized breakdown process (see Ref. 49, Fig. 14, p. 339). Further downstream, the amplitude of the asymmetric mode for case Low decays and $n=4$ is found to contain most energy at $z / r_{0}=9$ for all cases. At the last investigated axial position, we observe a broadbanded energy distribution and for wavenumbers $n \geq 16$ a drop-off of about two orders of magnitude.

As noted above, the eigenfunctions employed in the forcing reach their maxima in the shear layer of the baseflow profile. Thus, the effects of changes in forcing are expected to be most pronounced in the quantities along the jet nozzle lip line $r / r_{0}=1$, on which we will focus in the following. The streamwise development of the axial and radial velocity fluctuations is given in Figs. 13(a) and 13(b), respectively. Consistent with the expectations the RMS of the axial velocity fluctuations varies greatly and, in addition, exhibits a pronounced dual-peak distribution. Even for a small disturbance amplitude, such as $1.5 \%$ in case Low, the axial distribution of $\left\langle w^{\prime \prime 2}\right\rangle^{1 / 2} / U_{j}$ starts to rise at the inflow plane and 
reaches a local maximum three nozzle diameters downstream, before attaining the global maximum at $z / r_{0} \approx 8$, i.e., upstream the end of the potential core. As before, increasing the forcing amplitude shifts the transition process upstream and amplifies the dual-peak structure for which the global maximum now occurs at the first streamwise position. This dual-peak distribution is most likely a result of the localization of vortex pairings ${ }^{50}$ at this particular axial position. This assumption is further supported by the following arguments: As could be observed in various quantities so far, an increased forcing amplitude shifts the development of the shear layer upstream but also significantly reduces the streamwise jitter of the shear layer rollup. As the computation of the mean quantities also involves an azimuthal average, the dual peaks are more pronounced for cases Med and High compared to case Low. Azimuthal averaging over a helical alignment tends to smear out gradients, whereas an axisymmetric orientation due to the localization tends to amplify the dual-peak structure. The overall streamwise evolution of simulation Low is closest to results by Bogey and Bailly, ${ }^{16}$ however, because of the differences in the inflow forcing procedure their result does not exhibit a second peak related to the localized vortex pairings. Similar variations of the centerline longitudinal fluctuation intensity can for example be found in the experimental work of Zaman and Hussain $^{50}$ where profiles exhibit strong dual-peak distributions [see, e.g., their Figs. 3(a) or 27, pp. 455 and 483]. There, vortex pairing in an axisymmetric jet flow under excitation has been investigated. For an excitation frequency corresponding to $\mathrm{St}=0.85$ a dual-peak distribution is established, characterized by a trough region with reduced fluctuations located at the nozzle lip line which extends toward the jet centerline.

In contrast to the axial velocity, the development of the radial RMS fluctuations remains almost unaffected by the changes of the inflow forcing. This is also in agreement with experimental results ${ }^{50}$ which document that radial RMS fluctuations of a jet with controlled vortex pairing exhibit less variation compared to the axial component. Again, a reduction in amplitude seems to slow down the streamwise evolution. Best agreement with the results by Bogey and Bailly is found again for simulation Low. For completeness, we point out, however, that the axial profiles for the radial velocity RMS are higher than observed experimentally (overestimated by $\sim 40 \%$, see Ref. 16), which is also related to the artificially increased initial momentum thickness addressed before.

The effect of the forcing on the development of the vorticity thickness $\delta_{\omega}$, determined by $\delta_{\omega}=U_{j} /\left(\max _{r}|\partial\langle w\rangle / \partial r|\right)$, is shown in Fig. 14. The initially laminar shear layer thicken further upstream when increasing the disturbance amplitude. The streamwise growth is almost linear once the spreading has started. The growth rates of the vorticity thickness given in Table II have been determined by a linear fit to the data within $6 \leq z / r_{0} \leq 10$. The predicted values of $d\left(\delta_{\omega} / r_{0}\right) / d z$ $\approx 0.25$ are higher than those observed experimentally (see summary of experimental values in Ref. 51, ranging from 0.112 to 0.218 ), but are again in agreement with the study by Bogey and Bailly. ${ }^{16}$ Also note that case High has a stepwise

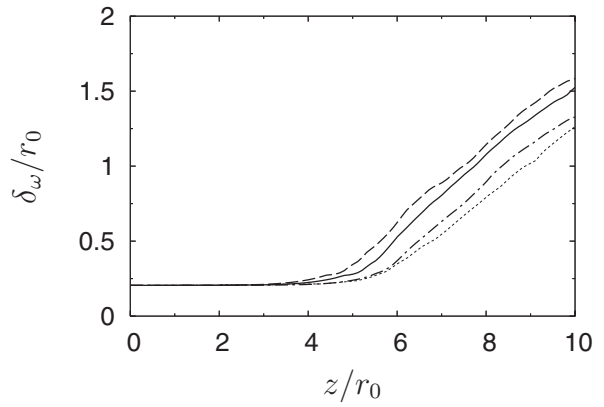

FIG. 14. Streamwise development of vorticity thickness $\delta_{\omega}:(-\cdot-)$ Low, (-) Med, (- - ) High, (. . ) Bogey and Bailly (Ref. 16).

increase in the vorticity thickness with a discontinuity around the location where the local minimum in the dualpeak distribution of the lip line RMS is observed.

The differences in the onset of the shear layer development also affect the turbulent stresses. This is exemplified by looking at the radial Reynolds normal stress and the shear stress in Figs. 15(a) and 15(b). The profiles are taken at equidistant streamwise locations $z / r_{0}=3,6$, and 9 and are shifted by the marked amount. Far upstream of the closing of the potential core the values rise rapidly, saturate within a distance of three radii, reach their maxima in the range $z / r_{0} \approx 5-6$ and decay in the streamwise direction. Corresponding to the previously noted occurrence of streamwiseelongated vortical structures further upstream for larger disturbance amplitudes, the peaks are higher at corresponding streamwise locations for higher forcing amplitudes. Downstream of $z / r_{0}=6$, which corresponds to the second profile in Fig. 15, the orientation changes and we now find case Low, whose streamwise development is delayed the strongest, to reach the largest Reynolds stress values. This very rapid development for case Low compared to the higher amplitude cases confirms the impression from the snapshots of instantaneous processes (see Figs. 5 and 6) that transition occurs in

(a)

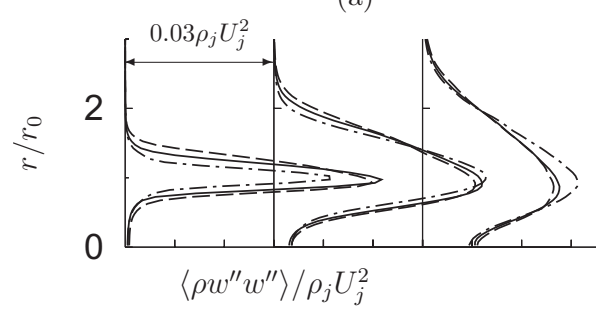

(b)

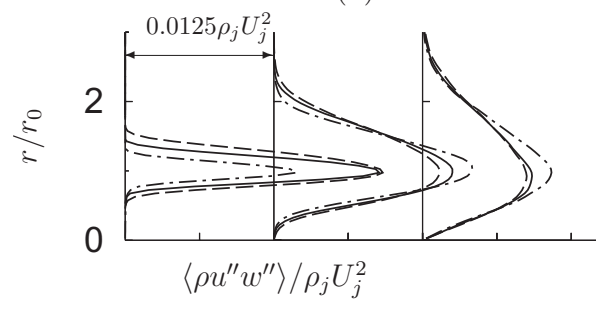

FIG. 15. Streamwise development of radial Reynolds stress profiles at positions $z / r_{0}=i \times 3 \quad\left(i=1, \ldots, 3\right.$ from left to right): (a) $\left\langle\rho w^{\prime \prime} w^{\prime \prime}\right\rangle / \rho_{j} U_{j}^{2}$, (b) $\left\langle\rho u^{\prime \prime} w^{\prime \prime}\right\rangle / \rho_{j} U_{j}^{2}:(-\cdot-)$ Low, (-) Med, and (-- ) High. 

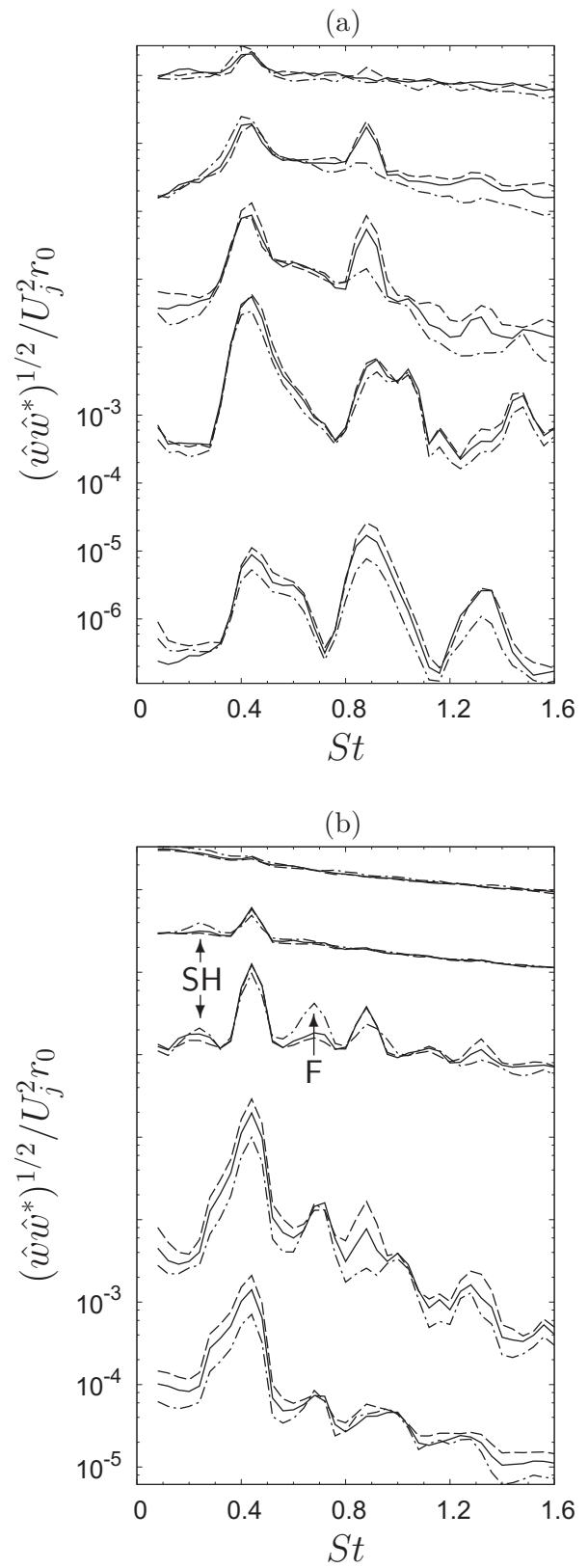

FIG. 16. Axial velocity spectra at streamwise positions $z / r_{0}=i \times 3$ $(i=0, \ldots, 4$ from bottom to top) along: (a) jet centerline, (b) jet lip line: (-.-) Low, (-) Med, and (- - ) High. The groups of spectra are shifted by a factor of 10 for clarity. Along the jet lip line $F$ denotes the occurrence of the fundamental frequency of unexcited mode $n=1, \mathrm{SH}$ the occurrence of its subharmonic.

a spatially more confined area and is more rapid. Also, as noted before, the process can be linked to the dominance of azimuthal wavenumbers $n=0$ and $n=1$ in this streamwise region as, for example, found in the one-dimensional turbulence kinetic energy spectra presented in Figs. 11 and 12.

This section is concluded by investigating the spectral composition of the axial velocity fluctuations along the jet centerline and the nozzle lip line. Nondimensional frequencies $\mathrm{St}=0.04$ are resolved in the spectra and we refer to Sec. II B for details concerning the data analysis. We focus on the transitional region of the jet flow and show the axial velocity spectra at different downstream positions in Fig. 16. The spectra at the inflow plane are as expected, i.e., the peak values along the jet centerline and the nozzle lip differ by two orders of magnitude [Fig. 16(a) versus Fig. 16(b)]. Highest peaks occur along the lip line where the magnitude of the axial velocity eigenfunction reaches its maximum and the alignment of the spectral amplitude follows the enforced disturbance amplitudes. In agreement with literature ${ }^{52}$ the flow is found to be most receptive to disturbances in a Strouhal number range around $\mathrm{St} \approx 0.4$, which corresponds to the column mode frequency ${ }^{50}$ (also referred to as preferred mode ${ }^{53}$ ). Overall, we observe a persistent and dominant existence of structures with this Strouhal number that are present along the centerline and the lip line. In combination with the TKE spectra shown in Figs. 11 and 12, we note that the eigenmode-based forcing does excite modes $n=4$ through $n$ $=8$ at the inflow, however, further downstream this interaction results in most-amplified structures with wavenumbers $n=0$ and $n=4$ at the frequency of $\mathrm{St}=0.43$. This Strouhal number corresponds to the excitation frequency of the mode $n=8$. A second dominant frequency, $\mathrm{St} \approx 0.88$, emerges at $z / r_{0}=3$ along the lip line presented in Fig. 16(b) and the three cases order according to their excitation amplitude. This frequency persists further downstream and at $z / r_{0}=6$ it appears at the jet centerline at $z / r_{0}=6,9$. It is important to note that at the centerline this peak at $\mathrm{St} \approx 0.88$ is observed only for cases Med and High and is not contained in the velocity signals of case Low.

At $z / r_{0}=6$ a pronounced subharmonic at $\mathrm{St} \approx 0.2$ emerges along the lip line, most obviously for the weakly disturbed flow. This subharmonic is marked by the symbol "SH" in Fig. 16(b). Its appearance is smeared out because the occurrence of the pairings with the fundamental frequency $\mathrm{St} \approx 0.43$ is random as pointed out before. For case Med, this subharmonic is less pronounced and decays much faster in the streamwise direction and in case High it appears only weakly. This supports the previously made observation that low-amplitude forcing results in a random but detectable vortex pairing and a persistent dominance of low frequencies $(\mathrm{St} \leq 0.5)$ downstream of $z / r_{0}=6$, whereas for case High a broader range of scales interact.

At $z / r_{0}=6$ case Low shows a distinct rise of a frequency around $\mathrm{St} \approx 0.7$ which is denoted by the symbol " $\mathrm{F}$ " in Fig. 16(b). This peak corresponds to the fundamental frequency of the asymmetric least stable mode $n=1$ that plays a dominant role in the transition process although it is initially unexcited (instantaneous data as well as TKE spectra, cf. Sec. III A). This frequency is in very good agreement with the linear stability theory, ${ }^{42,54}$ according to which the azimuthal wavenumbers $n=0,1$ reach their highest growth rates at the fundamental frequency of $\mathrm{St}=0.67$ and $\mathrm{St}=0.68$, respectively. Furthermore, with increasing distance from the orifice the ratio of the initial momentum thickness $\theta_{0}$ to jet radius $r_{0}$ increases and, hence, linear theory predicts a dominance of the helical wavenumber $n=1$ over the axisymmetric mode $n=0$.

In order to clarify the roles of the axisymmetric and the first helical shear layer instability modes during the onset of transition of case Low, the downstream development of the azimuthal Fourier amplitude $\hat{w}(n)$ is determined for two frequencies. The investigated fundamental frequencies of the 
(a)

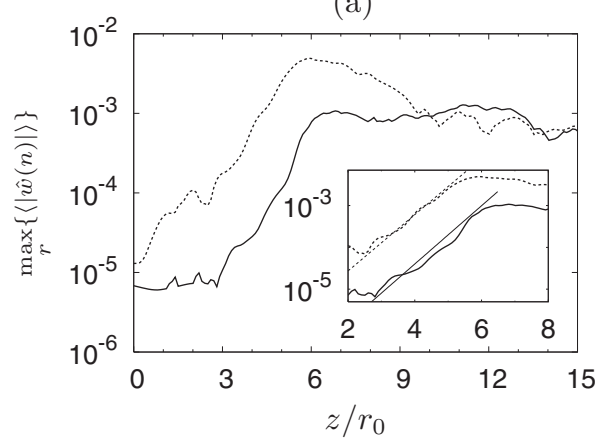

(b)

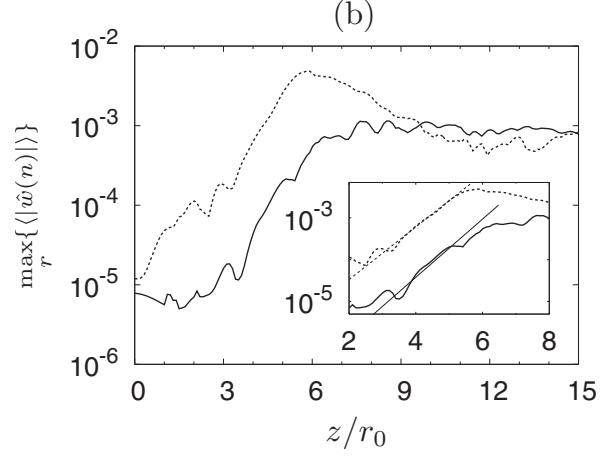

FIG. 17. Downstream development of Fourier modal amplitude $\hat{w}(n)$ of azimuthal wavenumbers (-) $n=0$ and ( . ) $n=1$ for (a) $\mathrm{St}_{0}^{n=0}=0.676$ and (b) $\mathrm{St}_{0}^{n=1}=0.681$. Inlay shows zoomed axial region $2 \leq z / r_{0} \leq 8$ together with straight lines denoting growth rates according to linear stability theory.

modes $n=0$ and $n=1$ correspond to the Strouhal numbers $\mathrm{St}_{0}^{n=0}=0.676$ and $\mathrm{St}_{0}^{n=1}=0.681$. According to LST the growth rates are $-\alpha_{i}^{n=0} r_{0}=1.60$ and $-\alpha_{i}^{n=1} r_{0}=1.55$, respectively. As the frequency difference between these two modes is very small the corresponding changes in growth rates are less than two decimal places. Figure 17 shows the downstream development of the modal amplitudes beyond the point of the closing of the potential core. For both frequencies, the modal amplitudes of the helical mode are significantly larger than the amplitude of the axisymmetric mode, in particular, around the relevant streamwise position $z / r_{0} \approx 6$. Thereby it is substantiated that the occurrence of the fundamental frequency can be linked to the helical azimuthal wavenumber $n=1$. As a side remark, we note that a very good agreement of the downstream development with the prediction of LST is observed for both modes shortly downstream of the inflow region, as can be seen in the inlays of Figs. 17(a) and 17(b).

\section{Acoustic results}

The three LES provide the directly computed jet noise in the acoustic near-field. These data allow to investigate the effect of the change in forcing amplitude on the pressure spectra, sound pressure levels, azimuthal two-point autocorrelations of the pressure fluctuations and, hopefully, allows to infer details of the underlying noise sources. To verify that hydrodynamic effects are separated from the acoustic regime, we follow Arndt et al. ${ }^{55}$ and choose an ad hoc threshold for $k \cdot r_{s} \leq 2$ where $r_{s}$ is the perpendicular distance from the jet centerline and $k$ the wavenumber given by $k=\omega / a_{\infty}$. This value has proven to be a reliable estimate ${ }^{56}$ and an approximate Strouhal number can readily be determined. The closest and most critical radial location to be analyzed in the following is $r_{s}=9 r_{0}$, for which $\mathrm{St}=k \cdot r_{s} r_{0} /\left(\pi \mathrm{Ma}_{j} r_{s}\right)$ $\leq 0.079$. For all radial locations, the estimated Strouhal numbers are therefore below $\mathrm{St}=0.1$ and the near-field pressure signals represent acoustic data.

In Fig. 18, the pressure spectra at different locations for the three investigated forcing amplitudes are shown. Recall that $\mathrm{St}=0.1$ is resolved in the analysis of the pressure spectra. For details of the time-signal processing we refer to Sec. II B. The end of the potential core of case Med is selected as the origin of a spherical coordinate system $(R, \theta, \phi)$. Three measurement points, with similar polar distances $R$ and polar angles that correspond to $\phi \approx 30^{\circ}, 60^{\circ}$, and $90^{\circ}$ measured from the positive jet axis $r=0$, are analyzed in Figs. 18 (a)-18(c). A close-up view of the range $0.6 \leq \mathrm{St} \leq 1.2$ can be found in graphs (d)-(f). The cylindrical and polar coordinates for the three observer locations are reported in Table III. Experimental reference data, shown by solid square symbols, are taken from a measurement campaign focusing on the spectral properties of noise in the acoustic near-field as well as the acoustic far-field ${ }^{57}$ of a $\mathrm{Ma}=0.9$ jet at $\mathrm{Re}=7.8$ $\times 10^{5}$. Dotted lines represent LES data reported in Ref. 58 . As tabulated in Table IV, the reference data are measured at slightly different polar angles and, more importantly, at different polar distances $R$ from the previously defined origin. Assuming a $1 / R$-decay for the pressure levels, a correction $\Delta_{\mathrm{SPL}}$ is determined with respect to the common polar distance $R^{*}=18 r_{0}$ and accounted for in all graphs. Note that the largest correction is $\Delta_{\mathrm{SPL}}=1.3 \mathrm{~dB}$ for the reference data and that the small differences in polar distances of the three cases Low, Med, and High are not accounted for. The spectra in Fig. 18 exhibit a strong dependence on the observation angle $\phi$, whereas the effect of the disturbance amplitude is noticed most dominantly at low frequencies and at intermediate observer angles. For small polar angles [Figs. 18(a) and 18(d)], the spectra are found in very good agreement, i.e., the spectral shape and also the peak of $125 \mathrm{~dB}$ is closely reproduced by our results which reach peak values between 124 and 127 $\mathrm{dB}$ depending on forcing amplitude. Our LES spectra are dominated by a frequency band below $\mathrm{St}=0.4$, which is the peak frequency for all cases. However, the experimentally determined peak of the spectra is around $\mathrm{St} \approx 0.2$ and the reference LES peak appears at a slightly higher Strouhal number, $\mathrm{St} \approx 0.3$. The spectrum for case Low exhibits more contributions at lower frequencies compared to the cases with higher excitation amplitudes. This enhanced lowfrequency band is related to the randomly occurring vortex pairings with helical wavenumber $n=1$ (see prominent rise of helical mode $n=1$ in Figs. 11, 12, and 17) whose fundamental frequency is halved to $\mathrm{St} \approx 0.35$ during the pairing. This kind of vortex pairings is known to efficiently contribute to noise emissions, in particular, in the downstream directions. ${ }^{3,59,60}$ Overall, case Low compares favorable with the reference LES. With increasing disturbance amplitude we observe less low-frequency noise, however, a tonal component around $\mathrm{St} \approx 0.9$ starts to appear which is most noticeable in the close-up provided in Fig. 18(d). The strength of the tonal peak is tied to less noise in the high-frequency range 
(a)

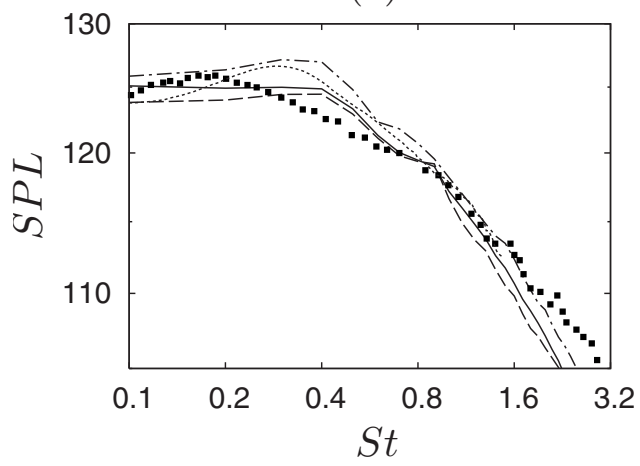

(b)

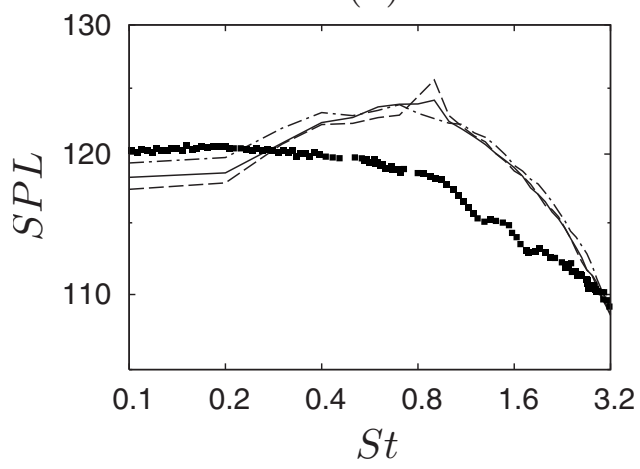

(c)

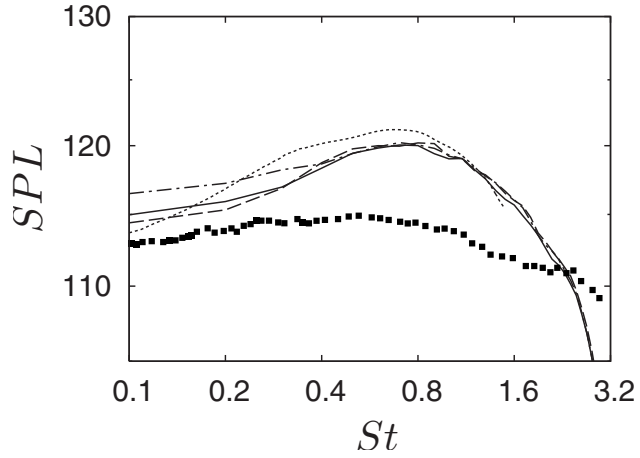

(d)

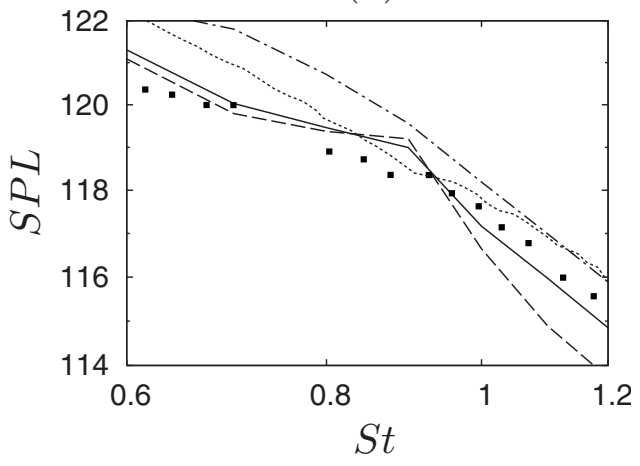

(e)

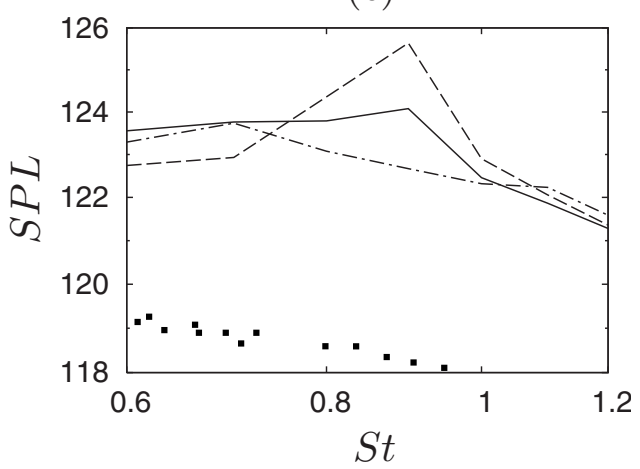

(f)

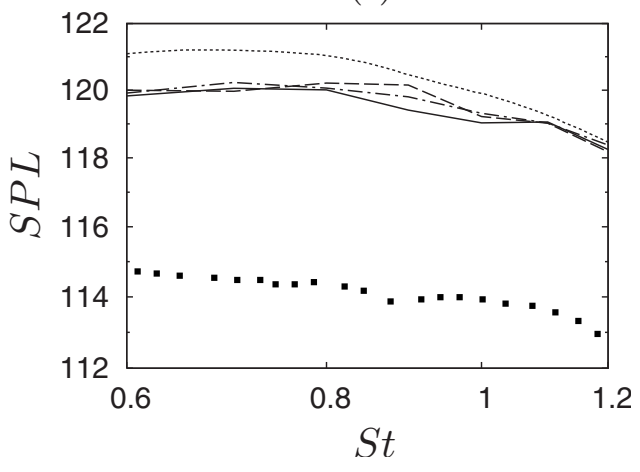

FIG. 18. Near-field pressure spectra in $\mathrm{dB}$ at (a) $\phi=30^{\circ}$, (b) $\phi=60^{\circ}$, and (c) $\phi=90^{\circ}$ : (-.-) Low, (-) Med, and (- - -) High. $(. \cdots)$ Bogey and Bailly (Ref. 58) at (a) $\phi=30^{\circ}$ and (c) $\phi=90^{\circ}$ only. Graphs (d) - (f) show close-up of frequencies $0.6 \leq \mathrm{St} \leq 1.2$

and, thus, the SPL drop-off sets in at lower frequencies for larger excitation levels.

At an increased observation angle, the overall dominance of the low frequencies is significantly reduced. In Figs. 18 (b) and $18(\mathrm{e})$, at $\phi \approx 60^{\circ}$, we find generally lower sound pressure levels for frequencies below $\mathrm{St}=0.4$, but again the induced large two-dimensional structures for case Low result in the highest amplitudes among the three investigated cases. For higher frequencies the experimental data are reproduced only qualitatively up to $\mathrm{St} \approx 2$. In the band $0.5 \leq \mathrm{St} \leq 2$ a large over prediction by approximately $5 \mathrm{~dB}$ is observed, whereas experiments yield $120 \mathrm{~dB}$. Again, cases Med and High exhibit a tonal component which is also the global maximum and, as before, its strength is positively correlated

TABLE III. Observer locations of spectra measurements and polar distances $R$ and angles $\phi_{\text {exact }}$ for all cases.

\begin{tabular}{|c|c|c|c|c|c|c|c|c|}
\hline \multirow[b]{2}{*}{$\phi$} & \multirow[b]{2}{*}{$r / r_{0}$} & \multirow[b]{2}{*}{$z / r_{0}$} & \multicolumn{3}{|c|}{$R / r_{0}$} & \multicolumn{3}{|c|}{$\phi_{\text {exact }}$} \\
\hline & & & Low & Med & High & Low & Med & High \\
\hline $30^{\circ}$ & 9 & 27 & 17.0 & 17.3 & 17.5 & $32.1^{\circ}$ & $31.3^{\circ}$ & $30.9^{\circ}$ \\
\hline $60^{\circ}$ & 15 & 21 & 17.2 & 17.4 & 17.5 & $60.8^{\circ}$ & $59.5^{\circ}$ & $59.0^{\circ}$ \\
\hline $90^{\circ}$ & 18 & 12 & 18.0 & 18.0 & 18.0 & $92.0^{\circ}$ & $90.5^{\circ}$ & $90.0^{\circ}$ \\
\hline
\end{tabular}


TABLE IV. Observer locations of spectra measurements and polar distances $R$ and angles $\phi_{\text {exact }}$ for reference data together with polar distance correction $\Delta_{\mathrm{SPL}}$ with respect to the common polar distance $R^{*}=18 r_{0}$.

\begin{tabular}{cccccccc}
\hline \hline$\phi$ & Ref. & $r / r_{0}$ & $z / r_{0}$ & $R / r_{0}$ & $\phi_{\text {exact }}$ & $R / R^{*}$ & $\Delta_{\mathrm{SPL}} / \mathrm{dB}$ \\
\hline $30^{\circ}$ & 57 & 15 & 30 & 23.3 & $40.1^{\circ}$ & 0.74 & +1.3 \\
& 58 & 12 & 29 & 20.7 & $35.5^{\circ}$ & 0.83 & +0.7 \\
$60^{\circ}$ & 57 & 15 & 20 & 16.9 & $62.4^{\circ}$ & 1.03 & -0.1 \\
$90^{\circ}$ & 57 & 15 & 10 & 15.2 & $98.2^{\circ}$ & 1.18 & -0.7 \\
& 58 & 15 & 11 & 15.0 & $94.5^{\circ}$ & 1.20 & -0.8 \\
\hline \hline
\end{tabular}

with the excitation level. The SPL peak of case High is very strong, $7 \mathrm{~dB}$ larger than the experimentally determined broadbanded spectrum, and still $3 \mathrm{~dB}$ larger than case Low. It is interesting to note that these peaks around $\mathrm{St}=0.9$ are outside of the range of initially excited frequencies but can equally be observed in the axial velocity spectra at the jet centerline and at the nozzle lip line (see Fig. 16).

In agreement with experimental as well as LES data, we find that at right angles to the jet axis the emitted noise is characterized by a broadband spectrum with no dominant frequencies, as can be seen for $\phi=90^{\circ}$ in Figs. 18(c) and 18(f). Again, we find significantly overpredicted sound pressure levels, i.e., the current simulations have a peak of 120 $\mathrm{dB}$ which is $6 \mathrm{~dB}$ higher compared to the experiments. ${ }^{57}$ For case Low a similar spectrum as in the reference LES is found but, as before, with a $2 \mathrm{~dB}$ enhancement in the low frequencies $\mathrm{St}<0.3$. All numerical results predict a slight shift of the peaks to higher Strouhal numbers, $\mathrm{St} \approx 0.8$ compared to $\mathrm{St} \approx 0.6$ in the experiments. The significantly overestimated pressure levels (in particular, in the sideline direction) might be related naturally to the increased radial RMS fluctuations observed before (see Sec. III B and Refs. 12, 16, and 58).

Overall, the main trends of the spectra provided by the simulations are in accordance with the reference data with a shift from a dominant low-frequency band at small angles from the jet axis to a broadband character in the sideline direction. However, the differences in the disturbance amplitude, and the associated changes in the transition process, generate a tonal peak around a Strouhal number of $\mathrm{St} \approx 0.9$ which is outside the band of excited frequencies. Nonlinear interaction of excited and initially unexcited eigenmodes, in connection with the preferred mode, might lead to the dominance of this frequency. This aspect, together with possible explanations, will be addressed in Sec. III C.

This section is concluded by a comparison of the azimuthal correlation properties of recorded pressure signals at two locations corresponding to the polar angles $\phi=30^{\circ}$ and $\phi=90^{\circ}$. The correlation coefficient is determined according to

$$
\mathcal{R}_{p p}(\Delta \theta)=\frac{\left\langle p^{\prime}\left(r, \theta_{0}, z, t\right) p^{\prime}\left(r, \theta_{0}+\Delta \theta, z, t\right)\right\rangle}{\left\langle p^{\prime 2}\left(r, \theta_{0}, z, t\right)\right\rangle^{1 / 2}\left\langle p^{\prime 2}\left(r, \theta_{0}+\Delta \theta, z, t\right)\right\rangle^{1 / 2}},
$$

using 5000 samples in time. At small angles from the jet axis, e.g., at $\phi=30^{\circ}$ as shown in Fig. 19(a), the pressure fluctuations for $\Delta \theta \leq 60^{\circ}$ are correlated similarly for the different forcing amplitudes and in accordance with the compu-
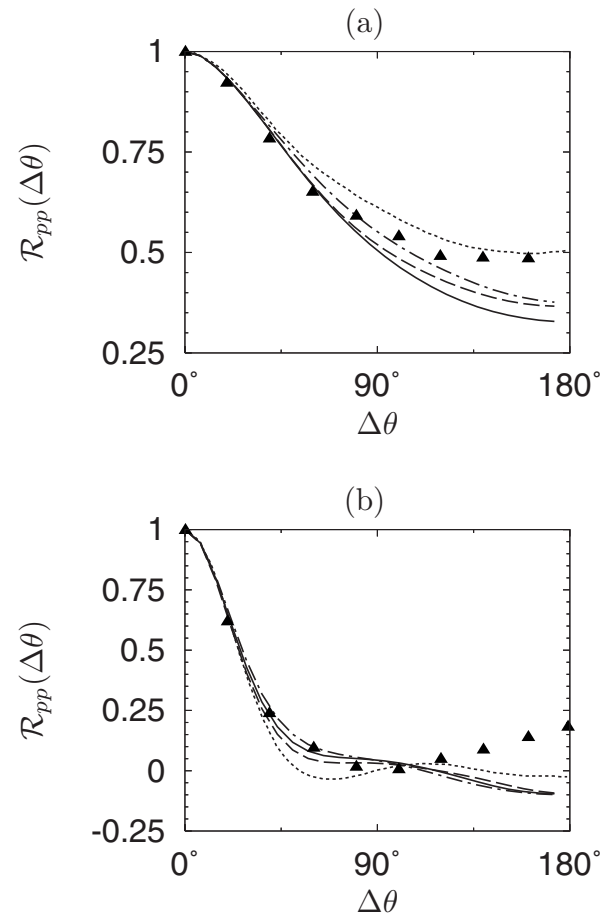

FIG. 19. Azimuthal correlation coefficient $\mathcal{R}_{p p}$ for pressure signals at (a) $\phi=30^{\circ}$ and (b) $\phi=90^{\circ}$ : (-.-) Low, (-) Med, and (- - ) High; (. .) Bogey and Bailly (Ref. 58); (ム) Maestrello (Ref. 61): (a) $\operatorname{Re}=5.2$ $\times 10^{4}, \mathrm{Ma}=0.88$ and (b) $\mathrm{Re}=4.4 \times 10^{4}, \mathrm{Ma}=0.74$.

tation by Bogey and Bailly ${ }^{58}$ (the locations of their measurements are given in Table IV) and the far-field experiments by Maestrello ${ }^{61}$ (taken at $R / r_{0} \approx 345$ ). For increasing azimuthal distances $\Delta \theta$, the correlations of cases Med and High decrease to $\mathcal{R}_{p p}\left(180^{\circ}\right) \approx 0.3$ and $\mathcal{R}_{p p}\left(180^{\circ}\right) \approx 0.35$, values that are clearly below the reference data that give $\mathcal{R}_{p p}\left(180^{\circ}\right)$ $\approx 0.5$. The correlation in this sector is slightly enhanced, $\mathcal{R}_{p p} \approx 0.4$, for case Low. Overall, the results for $\phi=30^{\circ}$ suggest a systematic lack of axisymmetric scales in the signal. Also, the naturally dominant helical mode that emerges during the early stages of transition in case Low (see onedimensional turbulence spectra, Figs. 11 and 12, and modal amplitude, Fig. 17) increases the correlations over all azimuthal distances. We also note that the ordering of the correlation coefficients for the three cases corresponds to the amount of turbulent kinetic energy contained in the low azimuthal wavenumbers $n=1$ and $n=2$, as shown in Fig. 12 . Again, this shows the emission efficiency of low azimuthal wavenumbers in the downstream direction. With respect to the pronounced reduction of coherence we note that Juvé et $a l .{ }^{62,63}$ reported correlation values for the unexcited jet of $\mathcal{R}_{p p}\left(180^{\circ}\right)=0.25$, which is in better agreement with our data. In addition, they find an increase to values in the range of the reference data when exciting the flow tonally at $\mathrm{St}=0.68$ (jet at $\operatorname{Re}=1.8 \times 10^{5}, \mathrm{Ma}=0.4$, and $\left.\mathcal{R}_{p p}\left(180^{\circ}\right) \approx 0.54\right)$.

The correlations for polar angle $\phi=90^{\circ}$ are shown in Fig. 19(b). At this location, similar results for all cases are found. For $\Delta \theta \geq 45^{\circ}$, the signals are almost uncorrelated, which is in agreement with the LES results by Bogey and 
TABLE V. Contributions to the acoustic field in downstream direction $\left(\phi=30^{\circ}\right)$ and sideline direction $\left(\phi=90^{\circ}\right)$.

\begin{tabular}{llllllllll}
\hline \hline$\phi$ & Case & $n=0$ & $n=1$ & $n=2$ & $n=3$ & $n=4$ & $n=5$ & $n=6$ & $n=7$ \\
\hline $30^{\circ}$ & Low & 0.56 & 0.31 & 0.08 & 0.03 & & & & \\
& Med & 0.52 & 0.35 & 0.08 & 0.03 & & & & \\
& High & 0.53 & 0.33 & 0.09 & 0.03 & & & & \\
& & & & & & & & & \\
$90^{\circ}$ & Low & 0.14 & 0.34 & 0.18 & 0.14 & 0.09 & 0.05 & 0.02 & 0.01 \\
& Med & 0.15 & 0.32 & 0.19 & 0.16 & 0.09 & 0.05 & 0.02 & 0.01 \\
& High & 0.14 & 0.30 & 0.20 & 0.17 & 0.09 & 0.05 & 0.02 & 0.01 \\
\hline \hline
\end{tabular}

Bailly $^{58}$ as well as with the far-field measurements by Maestrello $^{61}$ performed at a slightly lower Mach number of $\mathrm{Ma}=0.74$.

From the current findings for the azimuthal correlation we conclude that with increased amplitude the eigenmodebased inflow excitation of wavenumbers $n=4, \ldots, 8$ tends to break the symmetry with respect to the jet axis, especially at small angles from the jet axis. Note that the previously observed dominance of the helical wavenumber $n=4$ orders the structures in an axisymmetric fashion during transition but does not contribute to the correlation level at $\Delta \theta=180^{\circ}$. The reason for this loss of azimuthal coherence might be related to the transition process, which is characterized by the eigenmode interaction and the exclusion of low azimuthal wavenumbers from the inflow forcing, while exactly these wavenumbers are believed to constitute major sound sources in the downstream direction. ${ }^{58,61,62,64}$ Following Juvé et al., ${ }^{62}$ we employ an azimuthal Fourier expansion to obtain a more detailed picture of the different contributions of wavenumbers $n$ to the spatial correlation coefficient $\mathcal{R}_{p p}$ in the form of

$$
\mathcal{R}_{p p}(\Delta \theta)=\sum_{n=0}^{N_{\theta} / 2} a_{n} \cos (n \Delta \theta),
$$

where the sum of the coefficients $a_{n}$ is normalized to unity. The Fourier coefficients $a_{n}$ of mode $n$ with a contribution to the acoustic energy of more than $1 \%$ are reported in Table $\mathrm{V}$ for both observation angles $\phi$. As can be deduced from the presented correlations in the downstream direction, there is a reduced dominance of the axisymmetric mode $n=0$, but a significantly enhanced contribution from the helical wavenumber $n=1$ compared to Ref. 58 ( $\left.n=0,1: a_{n}=0.67,0.23\right)$. Again, we note that similar values to ours are reported in Ref. 63 and it is shown there that excitation increases the azimuthal correlation levels and accordingly the Fourier component $n=0$ at small angles. In the sideline direction, the Fourier coefficients are similarly reported in literature (see the work of Bogey and Bailly: ${ }^{58} n=0, \ldots, 3,: a_{n}=0.15,0.29$, 0.23 , and 0.19). However, the eigenmode forcing and the increased forcing amplitude seem to result in a transfer of energy from modes $n=2,3$, which are less energetic, to a broader range of wavenumbers $n \geq 5$.

\section{Links between jet flow transition and tonal near-field character}

In the remaining part, we attempt to establish a connection between the flow transition which is characterized by the eigenmode-based forcing and the tonal component observed in the near-field spectra at intermediate observer angles. By repeating the signal analysis with an enlarged subinterval length, the precise frequency of the tonal component is determined to be $\mathrm{St} \approx 0.876$. As pointed out before, the strength of the tone increases with disturbance amplitude and at the same time reduces the pressure levels over all other frequencies. This effect of turbulence and noise amplification as well as suppression is similar to effects investigated in a series of experiments by Zaman and co-workers. ${ }^{14,50,65}$ The tonal component could result from the breakdown process of structures that develop during the rollup of the initially laminar shear layers. The TKE analysis shows that the azimuthal wavenumber $n=4$ has direct relevance to the rollup and as this process proceeds its azimuthal higher harmonic $n=8 \mathrm{ab}$ sorbs significant amounts of turbulent kinetic energy. This can indicate a nonlinear interaction of structures described by the cut-and-connect process. The roles of interacting modes can further be clarified by analyzing their Fourier mode amplitudes $\hat{w}(n)$ at particular frequencies. We investigate the tone frequency $\mathrm{St}=0.876$ and its harmonics. Because of the dominance of $\mathrm{St} \approx 0.43$ in the axial velocity spectra we refer to this frequency as the fundamental $\mathrm{St}_{0}^{p}=0.438$, and thus its first harmonic frequency, $\mathrm{St}_{1}^{p}=2 \mathrm{St}_{0}^{p}$, corresponds to the peak in the near-field spectra. For clarity, we restrict the presentation to these two relevant frequencies and the modes that prevail during the transition process, i.e., modes $n=0,1,4$, and 8. Regardless of the excitation amplitude, shown in rows (a)-(c) of Fig. 20 for cases Low, Med, and High, similar downstream developments of the modal amplitudes $\hat{w}(n)$ are observed for the fundamental frequency $\mathrm{St}_{0}^{p}$ shown in column (A) and the first harmonic frequency $\mathrm{St}_{1}^{p}$ presented in column (B). Overall, the largest Fourier amplitudes occur at the fundamental frequency $\mathrm{St}_{0}^{p}=0.438$ for the wavenumber $n=4$, as can be seen in Fig. 20(a). Mode $n=4$ surmounts the complete transitional region with a peak around $z / r_{0} \approx 5$, followed by a slow downstream decay that lasts up to the potential core collapse. Consistent with our previous observations, the peak location shifts slightly upstream with increasing forcing amplitude. The varicose mode $n=0$ shortly rises close to the inflow and then saturates at a Fourier amplitude which is lower by one order of magnitude. As shown in Fig. 20(b), the analysis for $\mathrm{St}_{1}^{p}=0.876$ indicates that there are two relevant modes, $n=0$ and $n=8$, which shape the breakdown process. Their modal amplitudes are lower compared to the fundamental frequency and right from the inflow the axisymmetric mode plays a prominent role and rises almost linearly downstream of $z / r_{0} \approx 2$. Its importance is slightly reduced around $z / r_{0} \approx 6$, as can be seen from a small dip in $\hat{w}$ which enlarges with amplitude. This location, where $n=8$ has reached appreciable amplitude levels, coincides with the position where the dominant mode $n=4$ at $\mathrm{St}_{0}^{p}=0.438$ saturates. Thus, mode $n=4$ saturates and its azimuthal higher harmonic $n=8$, together with $n=0$, rapidly rises at the first harmonic fre- 
(A)

(a)

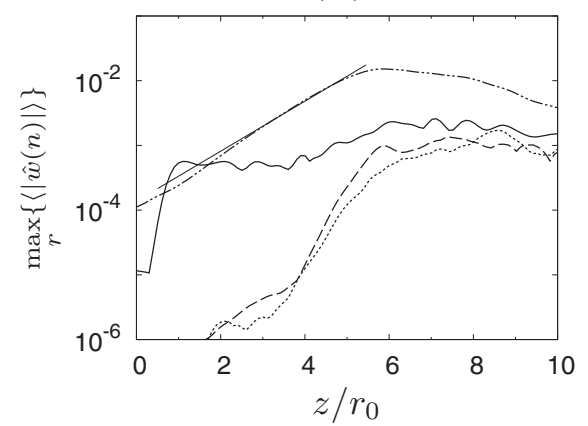

(b)

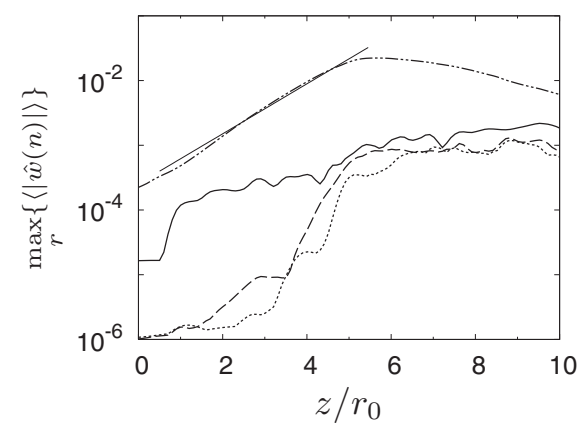

(c)

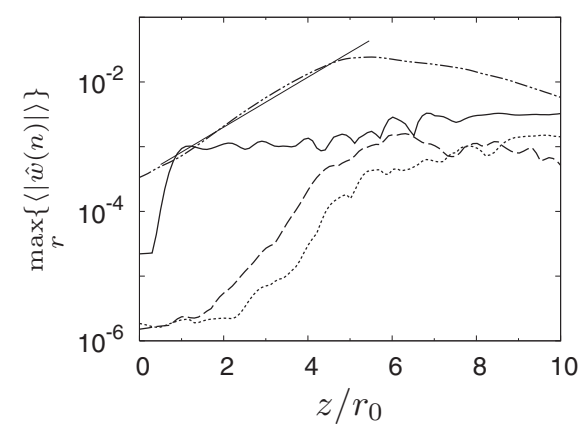

(B)
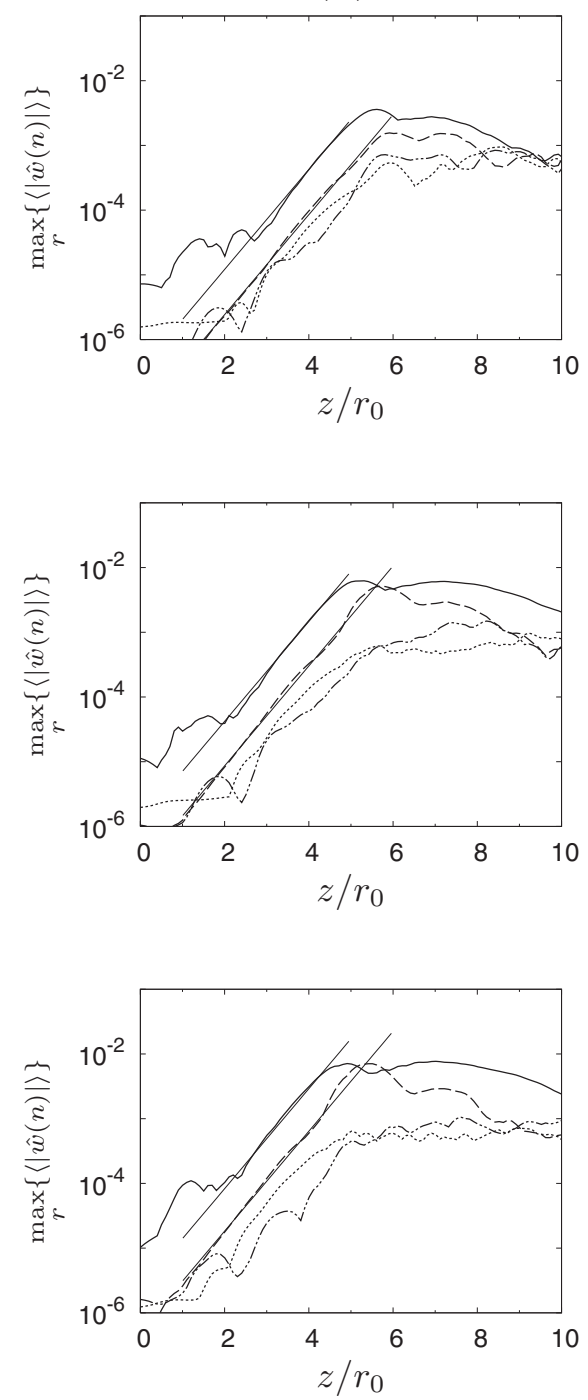

FIG. 20. Downstream development of Fourier mode amplitude $\hat{w}(n)$ of azimuthal wavenumbers $(-) n=0,(\cdots) n=1,(-\cdots-) n=4$, and $(---) n=8$ : (a) Low, (b) Med, and (c) High for (A) fundamental frequency $\mathrm{St}_{0}^{p}$ and (B) peak frequency corresponding to higher harmonic St ${ }_{1}^{p}$. Growth rates denoted by straight lines in row (A) according to linear theory and in row (B) according to weakly nonlinear theory.

quency. Exactly at the same downstream location the Reynolds stresses, presented in Fig. 15, saturate as well. Particularly for high forcing levels, the modal amplitude $\hat{w}(n=8)$ at $\mathrm{St}_{1}^{p}=0.876$ surpasses the axisymmetric mode at $z / r_{0} \approx 5.5$. Further downstream, the amplitude of $n=8$ is quickly reduced and the axisymmetric mode reassumes its dominance. The other azimuthal wavenumbers are found not to contribute to the overall process in a significant manner and behave similarly as mode $n=1$, i.e., at the two presented frequencies all other modes rise in a region between $2 \lesssim z / r_{0} \lesssim 5$ and then saturate at levels which are almost one order of magnitude below the relevant modes.

The interaction conditions, ${ }^{66}$ according to which waves are prone to subharmonic excitation when similar phase speeds occur, are not satisfied as instability waves $n=4$ and $n=8$ have drastically different phase speeds at $\mathrm{St} \approx 0.43$ (see Fig. 4). However, weakly nonlinear theory, as successfully applied by Sandham and Salgado, ${ }^{21}$ can explain this mode interaction. The frequency of the preferred mode seems to be
$\mathrm{St} \approx 0.43$ in our case because we force $n=8$ at exactly this frequency $(n= \pm 8$ are excited at $S t=0.43)$. Therefore, mode $n=4$ that initially is excited at $\mathrm{St}=0.63$ also picks up this particular frequency and grows. According to linear stability theory, mode $n=4$ has larger growth rates compared to its azimuthal harmonic $n=8$, which explains the dominance of $n=4$ at $\mathrm{St}_{0}^{p}=0.438$. The nonlinear interaction of two waves with wavenumber $n=4$ and frequency $\mathrm{St}_{1}^{p}=0.438$ generates azimuthal wavenumbers $n=0$ and $n=8$ at the tone frequency $\mathrm{St}_{1}^{p}$. The growth rate of mode $n=4$ according to LST is $-\alpha_{i}^{n=4} r_{0}=0.889$ and the nonlinearly excited modes $n=0$ and $n=4$ are found to grow at exactly twice this rate, i.e., $-\alpha_{i} r_{0} \approx 1.778$, which is included in the graphs for comparison. From our analysis of the Fourier modes we conclude that nonlinear interactions of wavenumbers $n=0,4$ and $n=8$ support the cut-and-connect process of toroidal structures.

The approach by Sandham and Salgado, ${ }^{21}$ which showed that driving an acoustic analogy with PSE-based mode-by- 
mode interactions can successfully model subsonic jet noise generation mechanisms, was adapted or simplified to our framework. Here, we apply the concept of weakly nonlinear mode-by-mode interaction to a high Reynolds number jet using eigenmodes determined from linear stability theory. These eigenmodes are determined assuming a parallel base flow and therefore neither the downstream development of the jet nor the interaction of the instabilities with the base flow are taken into account as done in the cases of linear/ nonlinear PSE. ${ }^{19,21}$ Nevertheless, the agreement between the growth rate determined using mode-by-mode interactions and the nonlinear simulation is striking and could help explaining an important subsonic noise generation mechanism observed in high Reynolds number jet flow. Further research in this area is necessary, as in the current study this nonlinear theory is employed only in order to clarify the origin of this tonal component in the near-field pressure spectra of the jet. To gain further insight into the particular transition mechanism and especially into the interaction of eigenmodes in the early nonlinear stages, a more detailed investigation of Fourier amplitudes in combination with PSE might be of interest. Clearly, this concept has to be addressed in more detail.

\section{CONCLUSIONS}

In this work results of three LES of a Ma $=0.9$ jet at $\mathrm{Re}=4.5 \times 10^{5}$ were presented, in which the focus is on the effect of inflow disturbances using a superposition of a hyperbolic-tangent base-flow profile and linearly unstable eigenmodes. The sensitivity of the simulation results to the physical disturbance generation has been investigated by varying the disturbance amplitude from $1.5 \%$ to $4.5 \%$ based on the jet exit velocity. The effects of unstable eigenmodes of azimuthal wavenumbers $|n|=4, \ldots, 8$ on the flow field development as well as on the directly computed near-field sound were found to be large, especially with respect to the transition process and the noise properties at certain observer angles.

With increasing forcing amplitude the transition process is shifted upstream as expected, which leads to an earlier onset of growing velocity fluctuations along the jet nozzle lip line and the jet centerline. As the forcing amplitude is increased the rollup process of the shear layers is modified. The analysis of the azimuthal mode dynamics shows that the large azimuthally coherent structures generated by lowamplitude excitation are dominated by the varicose and first helical mode $n=0$ and $n=1$. In contrast, the rollup process for high forcing amplitudes involves distorted threedimensional streamwise-elongated vortices dominated by the varicose mode $n=0$ and even modes $n=4$ and $n=8$. On the one hand, this amplitude-dependent modification of the rollup leads to a significant reduction of turbulence intensities along the jet centerline. The downstream development of the RMS fluctuations is found to be in good agreement with experimental as well as numerical data reported in the literature. On the other hand, the eigenmode forcing results in localized structures causing distinct dual-peak RMS distributions along the jet lip line as similarly observed in tonally forced jet experiments.
This modification of the transition process and the turbulence levels directly affects the acoustic near-field. As expected, the acoustic spectra significantly depend on the observer location. With increasing angle from the downstream jet axis, the dominance of a low-frequency band shifts from $\mathrm{St} \approx 0.4$ to higher frequencies around $\mathrm{St} \approx 0.9$. The strong emissions at Strouhal numbers $\mathrm{St} \leq 0.4$, in particular, for low $(1.5 \%)$ amplitude excitation, are linked to vortices that randomly undergo vortex pairings that most efficiently radiate in the downstream direction. These parings are caused by the initially unexcited, naturally least stable helical mode $n=1$. For medium (3\%) and especially for high (4.5\%) disturbance amplitudes, the pressure spectra contain a tonal component close to the higher harmonic of one of the forced fundamental frequencies as well as the frequency of the preferred (column) mode of the jet. The azimuthal correlation coefficients of the pressure fluctuations were determined for two polar angles $\phi=30^{\circ}$ and $\phi=90^{\circ}$ and support the established links. At small angles from the jet axis, the observed correlations are lower than the reference data, indicating less axisymmetric contributions by large, coherent structures to the pressure fluctuations. Experimental investigations of tonally excited jets, however, report similar distributions. In the sideline direction the signals are uncorrelated and match the data from literature, thereby supporting the established concept of noise emission associated with small-scale turbulence.

The analysis of Fourier mode amplitudes at the tone frequency and harmonics thereof shows that the tone observed in the near-field spectra can be linked to the rise, saturation and breakdown of structures that are generated during the early transition process. As a result of the excitation close to the preferred mode, the helical modes $n= \pm 4$ reach appreciable disturbance levels allowing its azimuthal higher harmonic $n=8$ as well as mode $n=0$ to rise with enhanced growth rates. These modes significantly contribute to noise emitted from a location slightly upstream of the collapse of the potential core which is perceived dominantly at intermediate observer locations. Supported by weakly nonlinear theory of eigenmode interaction this frequency can be explained as a result of an interaction of the axisymmetric mode and the initially excited modes $n= \pm 4$. The growth rates observed in the LES are found in very good agreement with estimates based on weakly nonlinear interactions of linearly unstable eigenmodes of the inflow profile.

\section{ACKNOWLEDGMENTS}

Funding by the Swiss National Science Foundation (SNF) is gratefully acknowledged. Computations were partially performed at the Swiss National Supercomputing Centre (CSCS) and partially carried out under the HPCEUROPA project (No. RII3-CT-2003-506079) with the support of the European Community-Research Infrastructure Action under the FP6 "Structuring the European Research Area" Program at the Höchstleistungsrechenzentrum Stuttgart (HLRS), Germany. We would like to thank C. Bailly for helpful discussions during a two-month research stay of F.K. at the Centre Acoustique, École Centrale de Lyon, France, and N. Sandham for sharing a draft of his 
(a)

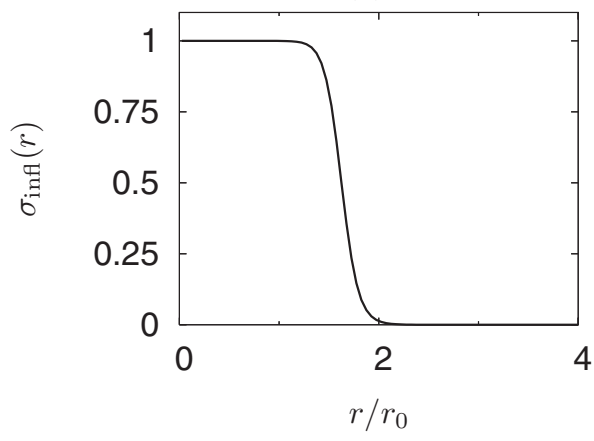

(b)

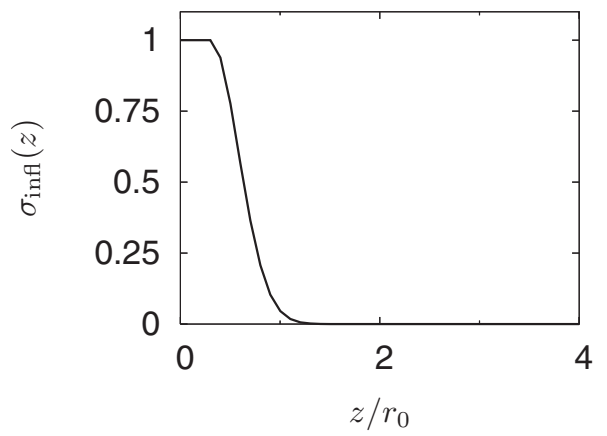

FIG. 21. Inflow sponge strength distribution: (a) $\sigma_{\text {infl }}(r, z)$ in radial direction for $z=0$ and (b) $\sigma_{\text {infl }}(r, z)$ in streamwise direction for first radial grid line $r=r_{\min }$

work concerning weakly nonlinear theory. We are grateful to Sebastian B. Müller, Andreas Jocksch, and Dominik Obrist for helpful discussions. We would also like to thank a referee for his comments that helped to improve the paper.

\section{APPENDIX A: IMPLEMENTATION OF DISTURBANCE TRIGGERING}

For completeness, we provide specific details of the inflow treatment below. When using a sponge zone, an artificial term is added to the right-hand side of the Navier-Stokes equations according to the general form,

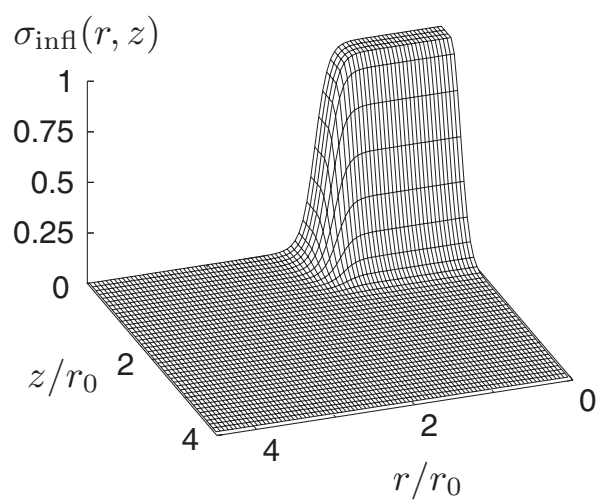

FIG. 22. Inflow sponge strength distribution viewing upstream: $\sigma_{\text {infl }}(r, z)$ in the vicinity of the inflow region.
TABLE VI. Description of random walk process with parameter bounds for $r n d_{1}$ and $r n d_{2}$.

\begin{tabular}{lcc}
\hline \hline Change by $\Delta$ & Range for $A_{n}$ & Range for $\phi_{n}$ \\
\hline Increase & $0.00<r n d_{1}<0.55$ & $0.00<r n d_{2}<0.65$ \\
No change & $\ldots$ & $0.65 \leq r n d_{2} \leq 0.85$ \\
Decrease & $0.55 \leq r n d_{1}<1.0$ & $0.85<r n d_{2}<1.0$ \\
\hline \hline
\end{tabular}

$$
\frac{\partial \mathbf{Q}}{\partial t}=\mathcal{R} \mathcal{H} \mathcal{S}(\mathbf{Q})-\sigma\left(\mathbf{Q}-\mathbf{Q}_{\mathrm{ref}}\right) .
$$

Here, $\mathbf{Q}$ denotes the unknown conservative state vector, $\mathcal{R H S}$ represents all terms of the Navier-Stokes equations written on the right-hand side, and $\mathbf{Q}_{\text {ref }}$ denotes a reference state to be defined. If the sponge coefficient $\sigma$ (that may vary in space and time) is greater than zero in Eq. (A1), the vector $\mathbf{Q}$ will be driven toward $\mathbf{Q}_{\text {ref }}$. As pointed out in Sec. II A (see Fig. 2), the disturbances introduced at the inflow are locally confined to the so-called inflow sponge. Its spatial extent is given by a combination of an exponential decay in the radial direction combined with a Gaussian-shaped profile in the downstream direction. This local confinement enables entrainment of the surrounding fluid. The functional form of this inflow sponge is given by

$\sigma_{\text {infl }}(r, z)=\left\{\begin{array}{rr}A_{\text {spg,infl }}[f(r) g(z)], & z>z_{0, \text { infl }}, \\ A_{\text {spg,infl }}\left[f(r) g\left(z_{0, \text { infl }}\right)\right], & 0 \leq z \leq z_{0, \text { infl }},\end{array}\right.$

where the inflow sponge amplitude $A_{\text {spg,infl }}=1.0$ and $f(r)$ and $g(z)$ are the radial and streamwise distributions defined as

$$
\begin{aligned}
& f(r)=\left(1+\left\{\exp \left[r^{2} \log \left(r_{c} / r_{0}\right)\right]-1\right\}^{n_{r}}\right)^{-1}, \\
& g(z)=\exp \left[-\left(\frac{z-z_{c, \text { infl }}}{d_{z_{\text {infl }}}}\right)^{2}\right] .
\end{aligned}
$$

In Eq. (A2), we set $z_{0, \text { infl }}=0.6 r_{0}$ which shifts the starting point of the semifinite Gaussian decay into the domain to have a short entrance region of constant sponge amplitude. Thus, for $z \leq z_{0 \text {,infl }}$ the sponge strength is set to $\sigma_{\text {infl }}(r, z)$ $=\sigma_{\text {infl }}\left(r, z_{0, \text { inf }}\right)$. The parameters determining the radial decay in Eq. (A3) are chosen as $r_{c}=1.3 r_{0}$ and $n_{r}=7.0$. The parameters of the Gaussian profile in Eq. (A4) are set to $z_{c, \text { infl }}=0.3 r_{0}$ and $d_{z_{\text {iffl }}}=0.4 r_{0}$. In Fig. 21(a) the radial dependence for this set of parameters is shown, whereas in Fig. 21(b) the streamwise profile is plotted. In Fig. 22 the sponge amplitude for the inflow plane is visualized as a threedimensional surface in the $r$-z-plane. In the inflow region this sponge is active, i.e., before the time advancement the conservative vector $\mathbf{Q}$ is driven toward the reference state $\mathbf{Q}_{\text {infl }}$. As described before (see Sec. II B), $\mathbf{Q}_{\text {infl }}$ is based on a col-

TABLE VII. Amount of change $\Delta$ used for inflow randomization of amplitude $A_{n}$ and phase $\phi_{n}$ and lower and upper bounds of amplitude $A_{n}$.

\begin{tabular}{lccc}
\hline \hline Quantity & $\Delta$ per time step & Min & Max \\
\hline$A_{n}$ & 0.005 & 0.03 & 0.10 \\
$\phi_{n}$ & $0.1 \omega_{n} \Delta t$ & $\cdots$ & $\cdots$ \\
\hline \hline
\end{tabular}


(a)

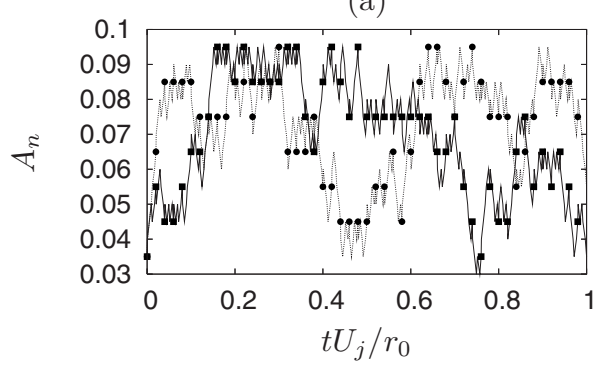

(b)

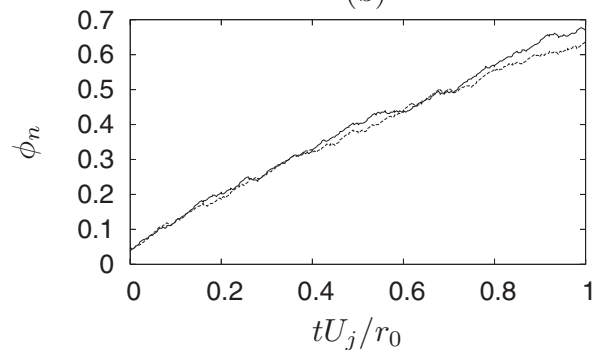

FIG. 23. Randomization of inflow disturbances: (a) disturbance amplitude $A_{n}$ and (b) phase shift $\phi_{n}$ for azimuthal wavenumbers $n= \pm 4 . n=4$ : (一) and $(\square) ; n=-4(-\ldots)$ and $(\bullet)$.

lection of instability waves that are superimposed on the laminar inflow profile [see Eq. (3)]. At each substep of the Runge-Kutta integration the amplitude $A_{n}$ and phase $\phi_{n}$ of each azimuthal modes $n$ are modified. We employ a similar random walk process as described by Lui ${ }^{67}$ but here, in addition to the phase, also modify the forcing amplitude. Therefore, random numbers $r n d_{1}$ and $r n d_{2}$ in the interval $r n d \in(0,1)$ are determined (using the random number generator described in Ref. 68, p. 272). Depending on the value of $r n d_{i}$, the amplitude and the phase are varied in a random walk process described in Table VI, i.e., increased or decreased by certain amounts $\Delta$ which are tabulated in Table VII. The combination of the chosen parameters results in a random distribution within the defined amplitude bounds, whereas the phase relations between various modes is continuously varied and according to the chosen values mostly increasing. Note that the amplitude of each mode $n$ is nonzero at all times as $A_{n} \in[0.03 ; 0.10]$ and that the maximum change per integration substep is restricted to $\Delta=0.005$ (see Table VII). For clarity this is exemplified by looking at the azimuthal wavenumber $n= \pm 4$ only. In Fig. 23(a) the temporal randomization of the disturbance ampli-

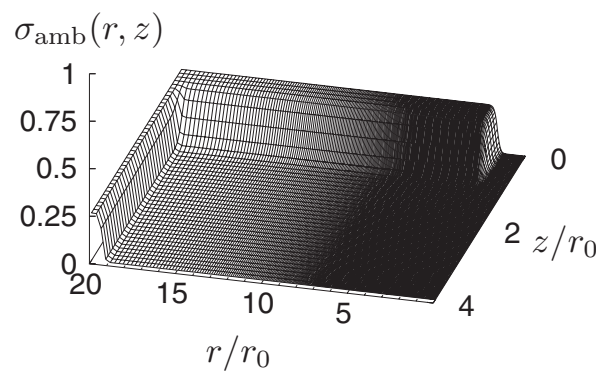

FIG. 24. Ambient sponge strength distribution: $\sigma_{\mathrm{amb}}(r, z)$ in the inflow region.

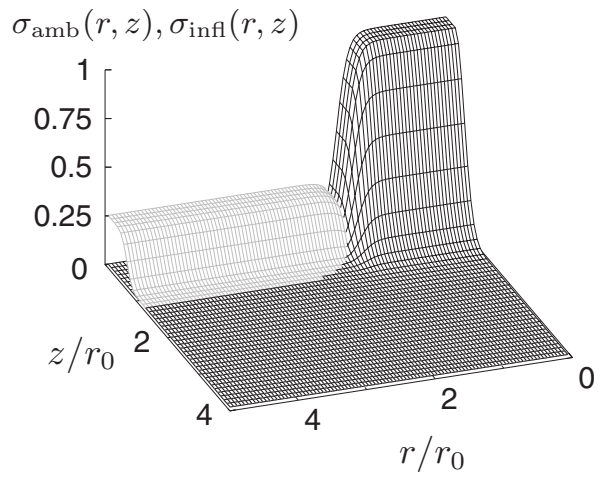

FIG. 25. Sponge amplitude distributions in vicinity of jet entrance: inflow sponge given in black, ambient sponge given in gray.

tude $A_{n}$ for azimuthal wavenumbers $n= \pm 4$ is shown along with the corresponding development of the phase shift $\phi_{n}$ in Fig. 23(b). The amplitude values for the full Runge-Kutta time steps (six-stage Runge-Kutta scheme) are marked by symbols. All modal excitation amplitudes $A_{n}$ are initialized using $A_{n}=\min \left(A_{n}\right)+\Delta A_{n}=0.035$ before starting the random walk process, but from thereon are independent of each other, as can be seen in Fig. 23(a).

\section{APPENDIX B: IMPLEMENTATION OF SPONGE LAYERS FOR PRESERVING AMBIENT STATE AND OUTFLOW DAMPING}

As described in Sec. II A, a drift of the mean pressure is prevented by employing a surrounding sponge layer that we refer to as ambient sponge. Similar to the inflow sponge, the conservative state vector $\mathbf{Q}$ is driven toward a state corresponding to ambient fluid at rest. However, in order not to prevent surrounding fluid from being entrained, only the density and energy (and hence pressure) are acted on. The spatial extent of the sponge layer is given by a combination of two error functions described by

$$
\sigma_{\mathrm{spg}, \mathrm{amb}}=A_{\mathrm{spg}, \mathrm{amb}}\left\{\sigma_{r}\left(1-\sigma_{z}\right)+\sigma_{z}[1-f(r)]\right\},
$$

where $A_{\mathrm{spg}, \mathrm{amb}}$ is set to $A_{\mathrm{spg}, \mathrm{amb}}=0.25$. The functions $\sigma$ for the radial and axial direction are given by the expressions

$$
\sigma_{r}=\frac{1}{2}\left(1-\operatorname{erf}\left\{\beta_{r}\left[-r+\left(r_{\max }-\frac{W_{r}}{2}\right)\right]\right\}\right),
$$

TABLE VIII. Parameters for inflow, ambient, and outflow sponge.

\begin{tabular}{lccc}
\hline \hline & $\begin{array}{c}\text { Inflow } \\
i=\text { infl }\end{array}$ & $\begin{array}{c}\text { Ambient } \\
i=\mathrm{amb}\end{array}$ & $\begin{array}{c}\text { Outflow } \\
i=\text { out }\end{array}$ \\
\hline$A_{i}$ & 1.0 & 0.25 & 1.0 \\
$\beta_{i}$ & $\beta_{r}=\beta_{z}=5.5$ & 0.5 \\
$W_{i}$ & & $W_{r}=1.2 ; W_{z}=1.25$ & 8.0 \\
$r_{\max }$ & $L_{r} / r_{0}$ & \\
$z_{\max }$ & & $L_{z} / r_{0}$ \\
\hline \hline
\end{tabular}




$$
\sigma_{z}=\frac{1}{2}\left\{1-\operatorname{erf}\left[\beta_{z}\left(z-\frac{W_{z}}{2}\right)\right]\right\},
$$

respectively. Here, $\beta_{i}$ denote the coefficients to adapt the steepness of the profile and $W_{i}$ is the corresponding spatial extents of the sponge layer, where the subscripts $r$ and $z$ denote the radial and axial directions. The algebraic formulation is as proposed by Bodony and Lele ${ }^{8}$ and Bodony. ${ }^{36}$ Similarly as the inflow sponge region is confined radially by $f(r)$ [Eq. (A3)], the ambient sponge starts just outside the inflow sponge by using the same function $f(r)$. The spatial distribution of the ambient sponge in the vicinity of the inflow region can be seen in Fig. 24. Figure 25 shows the inflow sponge and the ambient sponge in the jet entrance region.

The outflow sponge is defined similarly to the streamwise part of the ambient sponge,

$$
\sigma_{\text {out }}=\frac{A_{\text {spg,out }}}{2}\left(1-\operatorname{erf}\left\{\beta_{\text {out }}\left[-z+\left(z_{\text {max }}-\frac{W_{\text {out }}}{2}\right)\right]\right\}\right) .
$$

Here, $A_{\text {spg,out }}=1$ and denotes the amplitude at the outflow, $\beta_{\text {out }}$ is the coefficient to adapt the steepness of the profile and $W_{\text {out }}$ the corresponding spatial extent of the sponge layer. All sponge parameters are listed in Table VIII.

${ }^{1}$ M. J. Lighthill, "On sound generated aerodynamically. I. General theory," Proc. R. Soc. London, Ser. A 211, 564 (1952).

${ }^{2}$ J. E. Ffowcs Williams and D. L. Hawkings, "Sound generation by turbulence and surfaces in arbitrary motion," Philos. Trans. R. Soc. London, Ser. A 264, 321 (1969).

${ }^{3}$ T. Colonius, S. K. Lele, and P. Moin, "Sound generation in a mixing layer," J. Fluid Mech. 330, 375 (1997).

${ }^{4} \mathrm{~J}$. B. Freund, "Noise sources in a low-Reynolds-number turbulent jet at Mach 0.9," J. Fluid Mech. 438, 277 (2001).

${ }^{5}$ C. Bogey, C. Bailly, and D. Juvé, "Noise investigation of a high subsonic, moderate Reynolds number jet using a compressible large eddy simulation," Theor. Comput. Fluid Dyn. 16, 273 (2003).

${ }^{6} \mathrm{C}$. Bogey and C. Bailly, "Large eddy simulations of transitional round jets: Influence of the Reynolds number on flow development and energy dissipation," Phys. Fluids 18, 065101 (2006).

${ }^{7}$ D. J. Bodony and S. K. Lele, "Jet noise prediction of cold and hot subsonic jets using large-eddy simulation," Tenth AIAA/CEAS Aeroacoustics Conference and Exhibit, AIAA Paper No. 2004-3022, Manchester, 2004.

${ }^{8}$ D. J. Bodony and S. K. Lele, "On using large-eddy simulation for the prediction of noise from cold and heated turbulent jets," Phys. Fluids 17, 085103 (2005).

${ }^{9}$ A. Uzun, A. S. Lyrintzis, and G. A. Blaisdell, "Coupling of integral acoustics methods with LES for jet noise prediction," Int. J. Aeroacoust. 3, 297 (2004).

${ }^{10}$ N. Andersson, L.-E. Eriksson, and L. Davidson, "Investigation of an isothermal Mach 0.75 jet and its radiated sound using large-eddy simulation and Kirchhoff surface integration," Int. J. Heat Fluid Flow 26, 393 (2005).

${ }^{11}$ N. Andersson, L.-E. Eriksson, and L. Davidson, "Large-eddy simulation of subsonic turbulent jets and their radiated noise," AIAA J. 43, 1899 (2005).

${ }^{12}$ D. J. Bodony and S. K. Lele, "Current status of jet noise predictions using large-eddy simulation," AIAA J. 46, 364 (2008).

${ }^{13}$ C. A. Brown, "Acoustics of excited jets-a historical perspective," Technical Memorandum No. 213889, NASA-Glenn Research Center, 2005.

${ }^{14}$ K. B. M. Q. Zaman, "Far-field noise of a subsonic jet under controlled excitation," J. Fluid Mech. 152, 83 (1985).

${ }^{15}$ S. A. Stanley and S. Sarkar, "Influence of nozzle conditions and discrete forcing on turbulent planar jets," AIAA J. 38, 1615 (2000).

${ }^{16}$ C. Bogey and C. Bailly, "Effects of inflow conditions and forcing on subsonic jet flows and noise," AIAA J. 43, 1000 (2005).

${ }^{17}$ J. Laufer and T.-C. Yen, "Noise generation by a low-Mach-number jet," J. Fluid Mech. 134, 1 (1983).
${ }^{18}$ N. D. Sandham, C. L. Morfey, and Z. W. Hu, "Nonlinear mechanisms of sound generation in a perturbed parallel jet flow," J. Fluid Mech. 565, 1 (2006).

${ }^{19}$ L. C. Cheung, D. J. Bodony, and S. K. Lele, "Noise radiation predictions from jet instability waves using a hybrid nonlinear PSE-acoustic analogy approach," 13th AIAA/CEAS Aeroacoustics Conference and Exhibit, AIAA Paper No. 2007-3638, Rome, 2007.

${ }^{20}$ L. C. Cheung and S. K. Lele, "Aeroacoustic noise prediction and the dynamics of shear layers and jets using the nonlinear parabolized stability equations," Technical Report No. TF-103, Flow Physics and Computation Division, Stanford University, 2007.

${ }^{21}$ N. D. Sandham and A. M. Salgado, "Nonlinear interaction model of subsonic jet noise," Philos. Trans. R. Soc. London, Ser. A 366, 2745 (2008).

${ }^{22} \mathrm{~T}$. Suzuki and T. Colonius, "Instability waves in a subsonic round jet detected using a near-field phased microphone array," J. Fluid Mech. 565, 197 (2006)

${ }^{23}$ N. A. Adams, "Direct simulation of the turbulent boundary layer along a compression ramp at $M=3$ and $\operatorname{Re}_{\theta}=1685$," J. Fluid Mech. 420, 47 (2000).

${ }^{24} \mathrm{~K}$. Mohseni and T. Colonius, "Numerical treatment of polar coordinate singularities," J. Comput. Phys. 157, 787 (2000).

${ }^{25}$ J. B. Freund, P. Moin, and S. K. Lele, "Compressibility effects in a turbulent annular mixing layer," Technical Report No. TF-72, Flow Physics and Computation Division, Stanford University, 1997.

${ }^{26} \mathrm{~S}$. K. Lele, "Compact finite difference schemes with spectral-like resolution," J. Comput. Phys. 103, 16 (1992).

${ }^{27}$ A. Bayliss, A. Class, and B. J. Matkowsky, "Adaptive approximation of solutions to problems with multiple layers by Chebyshev pseudo-spectral methods," J. Comput. Phys. 116, 160 (1995).

${ }^{28}$ J. Berland, C. Bogey, and C. Bailly, "Low-dissipation and low-dispersion fourth-order Runge-Kutta algorithm," Comput. Fluids 35, 1459 (2006).

${ }^{29}$ S. Stolz, N. A. Adams, and L. Kleiser, "The approximate deconvolution model for large-eddy simulations of compressible flows and its application to shock-turbulent-boundary-layer interaction," Phys. Fluids 13, 2985 (2001).

${ }^{30}$ P. Schlatter, S. Stolz, and L. Kleiser, Direct and Large-Eddy Simulation V (Kluwer, Dordrecht, The Netherlands, 2004), pp. 65-72.

${ }^{31} \mathrm{P}$. Schlatter, "Large-eddy simulation of transition and turbulence in wallbounded shear flow," Ph.D. thesis, ETH Zürich, 2005.

${ }^{32}$ F. Keiderling, "Direct noise computation of high Reynolds number subsonic jet flow using LES," Ph.D. thesis, ETH Zürich, 2008.

${ }^{33} \mathrm{~K}$. W. Thompson, "Time dependent boundary conditions for hyperbolic systems," J. Comput. Phys. 68, 1 (1987).

${ }^{34}$ J. W. Kim and D. J. Lee, "Generalized characteristic boundary conditions for computational aeroacoustics," AIAA J. 38, 2040 (2000).

${ }^{35} \mathrm{M}$. Israeli and S. A. Orszag, "Approximation of radiation boundary conditions," J. Comput. Phys. 41, 115 (1981).

${ }^{36} \mathrm{D}$. J. Bodony, "Analysis of sponge zones for computational fluid mechanics," J. Comput. Phys. 212, 681 (2006).

${ }^{37}$ C. Lui and S. K. Lele, "Sound generation mechanism of shock-associated noise," Ninth AIAA/CEAS Aeroacoustics Conference and Exhibit, AIAA Paper No. 2003-3315, Hilton Head, SC, 2003.

${ }^{38}$ J. C. Lau, P. J. Morris, and M. J. Fisher, "Measurements in subsonic and supersonic free jets using a laser velocimeter," J. Fluid Mech. 93, 1 (1979).

${ }^{39}$ V. H. Arakeri, A. Krothapalli, V. Siddavaram, M. B. Alkislar, and L. M. Lourencoa, "On the use of microjets to suppress turbulence in a Mach 0.9 axisymmetric jet," J. Fluid Mech. 490, 75 (2003).

${ }^{40}$ K. Viswanathan, "Aeroacoustics of hot jets," J. Fluid Mech. 516, 39 (2004).

${ }^{41} \mathrm{C}$. Bogey and C. Bailly, "An analysis of the correlations between the turbulent flow and the sound pressure fields of subsonic jets," J. Fluid Mech. 583, 71 (2007).

${ }^{42}$ A. Michalke, "Survey on jet instability theory," Prog. Aerosp. Sci. 21, 159 (1984).

${ }^{43}$ S. B. Müller, F. Keiderling, and L. Kleiser, "Viscous compressible stability investigations in cylindrical coordinates," Proc. Appl. Math. Mech. 4, 468 (2004).

${ }^{44}$ S. B. Müller and L. Kleiser, "Viscous and inviscid spatial stability analysis of compressible swirling mixing layers," Phys. Fluids 20, 114103 (2008).

${ }^{45}$ D. J. Bodony and S. K. Lele, "Large eddy simulation of turbulent jets and progress towards a subgrid scale noise model," in Proceedings of International Workshop on "LES for Acoustics" (DLR, Göttingen, Germany, 2002). 
${ }^{46}$ J. Jeong and F. Hussain, "On the identification of a vortex," J. Fluid Mech. 285, 69 (1995).

${ }^{47}$ C. Bogey and C. Bailly, "Computation of a high Reynolds number jet and its radiated noise using large eddy simulation based on explicit filtering," Comput. Fluids 35, 1344 (2006).

${ }^{48} \mathrm{~J}$. C. Lau, "Effects of exit Mach number and temperature on mean-flow and turbulence characteristics in round jets," J. Fluid Mech. 105, 193 (1981).

${ }^{49}$ A. K. M. F. Hussain, “Coherent structures and turbulence," J. Fluid Mech. 173, 303 (1986)

${ }^{50}$ K. B. M. Q. Zaman and A. K. M. F. Hussain, "Vortex pairing in a circular jet under controlled excitation. Part 1. General jet response," J. Fluid Mech. 101, 449 (1980).

${ }^{51}$ E. Gutmark and C.-M. Ho, "Preferred modes and the spreading rates of jets," Phys. Fluids 26, 2932 (1983).

${ }^{52}$ P. Huerre and P. A. Monkewitz, "Local and global instabilities in spatially developing flows," Annu. Rev. Fluid Mech. 22, 473 (1990).

${ }^{53}$ S. C. Crow and F. H. Champagne, "Orderly structure in jet turbulence," J. Fluid Mech. 48, 547 (1971).

${ }^{54}$ A. Michalke and G. Hermann, "On the inviscid instability of a circular jet with external flow," J. Fluid Mech. 114, 343 (1982).

${ }^{55}$ R. E. A. Arndt, D. F. Long, and M. N. Glauser, "The proper orthogonal decomposition of pressure fluctuations surrounding a turbulent jet," J. Fluid Mech. 340, 1 (1997).

${ }^{56} \mathrm{C}$. E. Tinney and P. Jordan, "The near pressure field of co-axial subsonic jets," J. Fluid Mech. 611, 175 (2008).

${ }^{57}$ C. Bogey, S. Barré, V. Fleury, C. Bailly, and D. Juvé, "Experimental study of the spectral properties of near-field and far-field jet noise," Int. J. Aeroacoust. 6, 73 (2007).
${ }^{58} \mathrm{C}$. Bogey and C. Bailly, "Investigation of donwstream and sideline subsonic jet noise using large eddy simulation," Theor. Comput. Fluid Dyn. 20, 23 (2006).

${ }^{59}$ B. E. Mitchell, S. K. Lele, and P. Moin, "Direct computation of the sound generated by vortex pairing in an axisymmetric jet," J. Fluid Mech. 383, 113 (1999)

${ }^{60} \mathrm{C}$. Bogey, C. Bailly, and D. Juvé, "Numerical simulation of sound generated by vortex pairing in a mixing layer," AIAA J. 38, 2210 (2000).

${ }^{61}$ L. Maestrello, "Two-point correlations of sound pressure in the far field of a jet: experiment," Technical Memorandum No. 72835, NASA-Langley Research Center, 1976.

${ }^{62}$ D. Juvé, M. Sunyach, and G. Comte-Bellot, "Filtered azimuthal correlations in the acoustic far field of a subsonic jet," AIAA J. 17, 112 (1979).

${ }^{63}$ D. Juvé and M. Sunyach, "Near and far field azimuthal correlations for excited jets," Seventh AIAA Aeroacoustics Conference, AIAA Paper No. 1981-2011, Palo Alto, CA, 1981.

${ }^{64}$ A. Michalke and H. V. Fuchs, "On turbulence and noise of an axisymmetric shear flow," J. Fluid Mech. 70, 179 (1975)

${ }^{65}$ K. B. M. Q. Zaman and A. K. M. F. Hussain, "Turbulence suppression in free shear flows by controlled excitation," J. Fluid Mech. 103, 133 (1981).

${ }^{66}$ J. Cohen and I. Wygnanski, "The evolution of instabilities in the axisymmetric jet. Part 2. The flow resulting from the interaction between two waves," J. Fluid Mech. 176, 221 (1987).

${ }^{67}$ C. C. M. Lui, "A numerical investigation of shock-associated noise," Ph.D. thesis, Stanford University, 2003.

${ }^{68}$ W. H. Press, S. Teukolsky, T. M. Vetterling, and B. P. Flannery, Numerical Recipes in Fortran 77, 2nd ed. (Cambridge University Press, Cambridge, England, 1992). 